\title{
Effect of Aspect Ratio on Rigid Lifting Flat Plates in Pitch- Plunge Motion at Low Reynolds Numbers
}

\author{
Jonathan M. Rausch ${ }^{1}$, Yeon Sik Baik ${ }^{2}$, Lu is P. Bernal ${ }^{3 *}$ and Michael V. Ol ${ }^{4} * *$ \\ *University of Michigan, Ann Arbor, MI, 48109 \\ **U.S. Air Force Research Laboratory, Wright-Patterson Air Force Base, OH 45433, U.S.A.
}

\begin{abstract}
Flowfield behavior of wall-to-wall and low aspect ratio $(\mathrm{AR}=2)$ rigid flat plates in pitching and plunging motion is studied in a water tunnel at low Reynolds numbers. All models have a $152 \mathrm{~mm}$ chord and $\mathrm{t} / \mathrm{c}=\mathbf{2 . 2 5 \%}$. Two sets of kinematics already wellestablished in the recent literature are used: combined pitch and plunge, where pitch angle of attack partially cancels plunge-induced angle of attack, and pure plunge. Three Reynolds numbers $\left(1 \times 10^{4}, 3 \times 10^{4}\right.$, and $\left.6 \times 10^{4}\right)$ are considered, using dye flow visualization and particle image velocimetry (PIV). Broadly, sectional vorticity contour plots reveal similarity between the nominally $2 \mathrm{D}$ and $\mathrm{AR}=2$ plates, but there are several interesting exceptions. The $A R=2$ plate exhibited a marked axial flow when the effective angle of attack was near its maximum. For the pitching and pl unging motion the $A R=2$ plate showed open separation at phase of $180^{\circ}$, while the nominally two-dimensional model has closed separation throughout the downstroke. For validation of the PIV results, a comparison is made between Reynolds number $3 \times 10^{4}$ and $4 \times 10^{4}$ three-dimensional models at the Uni versity of Michigan and Air Force Research Laboratory (AFRL), respectively.
\end{abstract}

\section{Nomenclature}

c

$f$

$h_{o}$

$k$

$L$

Re

St

$t$

$t / c$

$\bar{u}$

$\bar{u}$

$\overline{u^{\prime} v}$

$U_{\infty}$

$\bar{v}$

$\bar{v}^{\prime}$

$x$

$\frac{x}{c}$

chord [m]

frequency [Hz]

reduced plunge amplitude of oscillation

reduced pitch frequency, $k=\pi f c / U_{\infty}$

length [m]

Reynolds number, $R e=c U_{\infty} / v$

Strouhal number, $S t=2 f h_{o} / U_{\infty}=2 k h_{o} / \pi$

time [s]

thickness-to-chord ratio [\%]

mean $x$-component of velocity vector $[\mathrm{m} / \mathrm{s}$ ]

mean fluctuation of $x$-component of velocity vector $[\mathrm{m} / \mathrm{s}]$

Reynolds stress $\left[\mathrm{m}^{2} / \mathrm{s}^{2}\right]$

freestream velocity $[\mathrm{m} / \mathrm{s}$ ]

mean $y$-component of velocity vector $[\mathrm{m} / \mathrm{s}]$

mean fluctuation of $y$-component of velocity vector $[\mathrm{m} / \mathrm{s}]$

Cartesian $x$-coordinate [m]

chord normalized $x$-coordinate

\footnotetext{
${ }^{1}$ Graduate Research Assistant, University of Michigan, Department of Aerospace Engineering

${ }^{2}$ Graduate Research Assistant, University of Michigan, Depart ment of Aerospace Engineering

${ }^{3}$ Associate Profes sor, University of Michigan, Depart ment of Aerospace Engineering, lpb@u mich.edu.

${ }^{4}$ Aerospace Engineer, Air Vehicles Directorate, Wright-Patters on AFB
} 


$\begin{array}{ll}y & \text { Cartesian } y \text {-coordinate }[\mathrm{m}] \\ \frac{y}{c} & \text { chord normalized } y \text {-coordinate }\end{array}$

\section{Greek Sy mbols}

$\begin{array}{ll}\alpha_{e f f} & \text { effective angle of attack [deg.] } \\ \alpha_{0} & \text { mean angle of attack [deg.] } \\ \lambda & \text { ratio of pitch a mplitude to plunge induced angle of attack a mplitude } \\ v & \text { kinematic viscosity }\left[\mathrm{m}^{2} / \mathrm{s}\right] \\ \phi & \text { positive phase offset for pitch relative to plunge [rad.] or phase of motion [deg.] } \\ \omega^{*} & \text { normalized vort icity, } \omega^{*}=\omega c / U_{\infty}\end{array}$

\section{Introduction}

We continue our series of water tunnel experiments on the flowfields of pitching and plunging rigid foils and plates, now with extension to low aspect ratio planforms. The motivating application continues to be Micro Air Vehic les (MAVs) ${ }^{1}$ and the resulting low Reynolds numbers $\left(10^{4}-10^{5}\right)$. The roles of flow separation and large scale vortical structures in flapping wing flight are of particular interest. The kinematics of flapping wing flight involve large amplitude oscillations of pitch and plunge. The presence of flow separation and large scale vortical structures has notional parallels to dynamic stall ${ }^{2}$ phenomena generally associated with helicopter blade research ${ }^{3}$. Dynamic stall is the formation, separation, and convection of a leading edge vortex that produces a transient chordwise pressure distribution significantly different from static stall. In addition to the physics associated with dynamic stall phenomena, the role of laminar-to-turbulent transition in flapping wing flight remains of significance, since transition can significantly affect the nature of flow separation and thus the aerodynamic loads time history ${ }^{4}$.

Here we extend recent work on wall-to-wall or nominally 2D pitching/plunging plates to low aspect ratio. Baik, et $a l{ }^{4}$ examined the effect of Reynolds number and airfoil shape for nominally two-dimensional models. We retain the same kinematics, but generalize to an $A R=2$ plate, and consider second-order flowfield statistics for the $2 \mathrm{D}$ and low-A R case in detail.

\section{Expe rimental Setup}

The University of Michigan's closed loop, free surface water tunnel has test section of 61 x $61 \mathrm{~cm}$ with glass sides and bottom, freestream velocity range from $5 \mathrm{~cm} / \mathrm{s}$ to $40 \mathrm{~cm} / \mathrm{s}$, and turbulence intensity of $1.15 \%$ (measured using particle image velocimetry, and including low-frequency bulk-flow undulations).

Models are actuated by a pitch-plunge oscillator rig above the tunnel's free surface, consisting of a B4872TS Rotary Table (for pitch) and a Velmex 20-inch BiSlide (for plunge). Both motors are controlled by a Velmex VXM1-1 motor controller. Models are suspended vertically from the pitch-plunge oscillator rig. Both models were stainless steel with a $t / c=2.25 \%$ and $c=152 \mathrm{~mm}$. For clarification the two flat plate models will be refe rred to as the " $2 \mathrm{D}$ model" for the model spanning the test section and the " $3 \mathrm{D}$ model" for the AR=2 model. The $3 \mathrm{D}$ model is an $\mathrm{AR}=1$ semi-span with an endplate used as a symmetry plane. The surfaces of the stainless steel flat plates were polished for a specular reflection of PIV laser light.

Dye injection was used for qualitative flow visualization and to predicatively arrange the PIV. Dye is introduced by a seven-prong rake upstream of the model at the midspan for the $2 \mathrm{D}$ plate and at the 3/4-span for the 3D plate, and is driven by a positive-displacement infusion pump to maintain efflux speed matching with the free-stream. The resulting streaklines produce a global portrait of flow separation and if interpreted with care, are a useful surrogate for tagging vortical structures. Spanwise locations of dye injection also corres pond to the PIV measurement planes.

Two camera views were used to capture the flow visualization. One was perpendicular to plane of the injected dye flow and is thus coincident with PIV. The other was normal to the free-stream, to capture spanwise flow.

\section{A. Particle Image Velocimetry}

The PIV system is double frame, single exposure, using a Quanta-Ray PIV Series Pulsed Nd:YAG laser and two Cooke Corporation PCO.4000, 4008 x 2672 pixel CCD cameras. Both cameras used Nikon 105 mm lenses. Figure 1a depicts the orientation of the laser light sheet and camera relative to the test section of the water channel. Figure $1 \mathrm{~b}$ is dye flow visualization image for the $3 \mathrm{D}$ model. In the image the coincident PIV/dye-flow plane is marked at the 3/4-span location with the green line; additionally, the end plate is observed at the top of Figure 1b. Table 1 recites the various PIV operational parameters. 


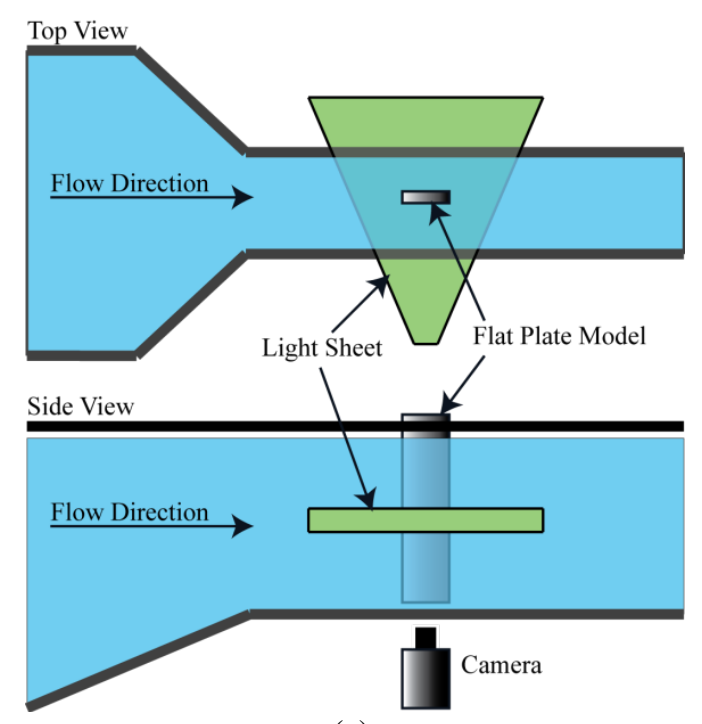

(a)

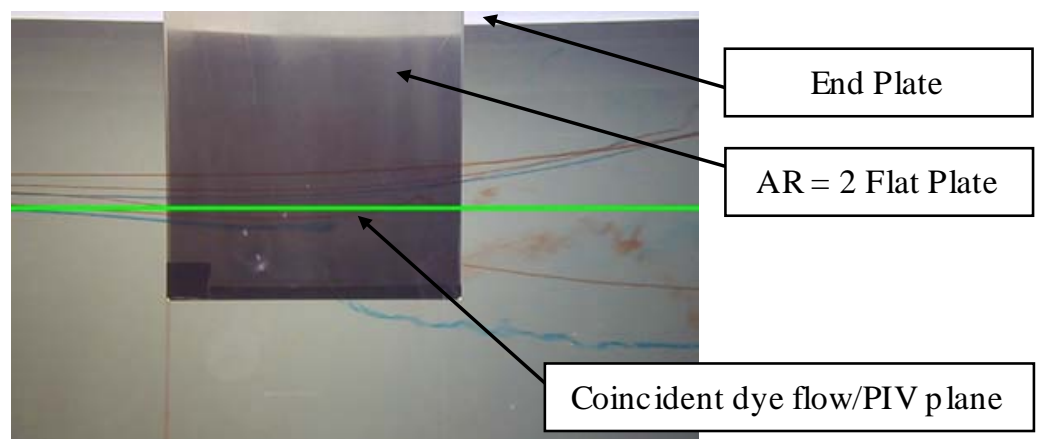

(b)

Figure 1. Diagram of the PIV setup with respect to the water channel's test section. The diagram is an example of the two-dimensional flat plate setup with the model spanning the entire depth of the test section. 
Table 1. Table of PIV parameters for the two- and three-dimensional flat plates

\begin{tabular}{|c|c|}
\hline \multicolumn{2}{|l|}{ Model } \\
\hline Chord, c [mm] & 152 (for 2D and 3D models) \\
\hline t/c ratio $[\%]$ & 2.25 \\
\hline 3D flat plate length $[\mathrm{mm}]$ & 152 \\
\hline \multicolumn{2}{|l|}{ Flow } \\
\hline Fluid & Water \\
\hline $\operatorname{Re}$ & $1 \times 10^{4}, 3 \times 10^{4}$, and $6 \times 10^{4}$ \\
\hline$U_{\infty}[\mathrm{cm} / \mathrm{s}]$ & $6.6,19.7$, and 39.5 (in order of increasing $\mathrm{Re}$ ) \\
\hline \multicolumn{2}{|l|}{ Seeding } \\
\hline Type & $\mathrm{TiO}_{2}$ \\
\hline Particle Phy sical Diameter [ $\mu \mathrm{m}]$ & 3 \\
\hline Dispersant & DARVAN C-N \\
\hline \multicolumn{2}{|l|}{ Laser } \\
\hline Type & Nd:YAG \\
\hline Max Pulse Energy [mJ/pulse] & 200 \\
\hline \multicolumn{2}{|l|}{ Recording } \\
\hline approx. FOV $\left[\mathrm{mm}^{2}\right]$ & 20,000 \\
\hline Camera & PCO. 4000 \\
\hline Pixel Length Scale [pixels/mm] & 25 \\
\hline Dy namic Range & 16 bit \\
\hline f-number & 5 \\
\hline Focal length [mm] & 105 \\
\hline Particle Image Diameter [pixels] & 3 \\
\hline$\Delta t[\mathrm{~ms}]$ & $4.82,1.60$, and 0.80 (in order of increasing Re) \\
\hline \multicolumn{2}{|l|}{ Image Pair Processing } \\
\hline High Resolution Window [pixels ${ }^{2}$ ] & $32 \times 32$ \\
\hline Low Resolution Window [pixels ${ }^{2}$ ] & $64 \times 64$ \\
\hline Sample Size* [min. - max.] & approx. $175-200$ \\
\hline Total DPIV Realizations & 200 \\
\hline
\end{tabular}

\section{B. Air Force Research Labor atory Experiments}

Experiments performed in the AFRL Horizontal Free-surface Water Tunnel (HFWT) on a very similar flow configuration are also reported here. The HFWT has a $46 \mathrm{~cm}$ wide by $61 \mathrm{~cm}$ deep test section with glass sides and bottom, freestream velocity range from $3 \mathrm{~cm} / \mathrm{s}$ to $45 \mathrm{~cm} / \mathrm{s}$, and turbulence intensity of $0.5 \%$. The each plunge rod is attached to a linear servomotor oriented vertically above the test section of the HFWT. For a more detailed discussion of the experimental facility and PIV procedure consult $\mathrm{Ol}$, et al. ${ }^{2}$

In contrast to the model mounting scheme used at the University of Michigan, the AFRL model is a full span model mounted on a sting attached to the model's trailing edge at the midspan. The moutning scheme is depicted in Figure 2.

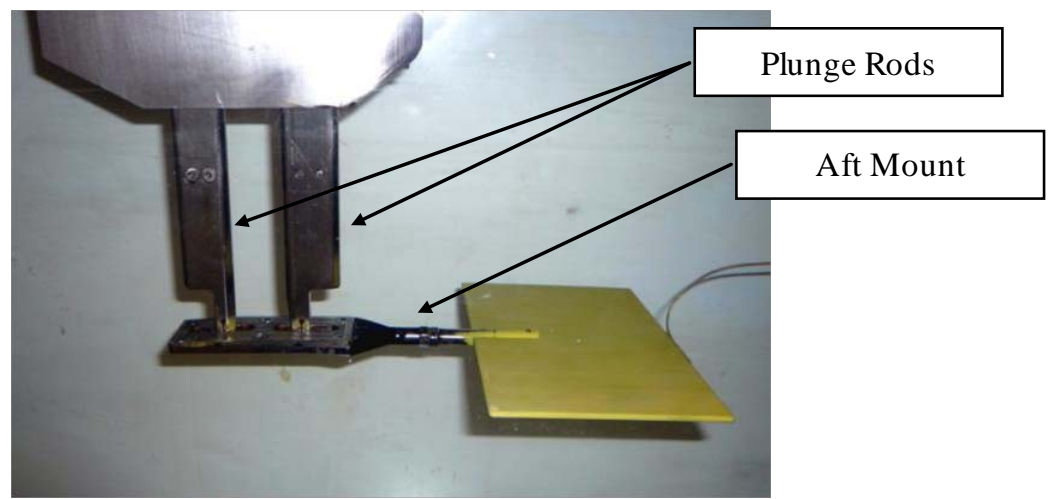

Figure 2. AFRL aft sting mount

4

American Institute of Aeronautics and Astronautics 
In the AFRL experiments the kinematic scaling parameters are the same as the University of Michigan (UM) experiments except for the Reynolds number. The AFRL data has Reynolds number 40,000 while the UM data has Reynolds number 30,000.

\section{Results}

The two model kinematics are a pitch--plunge and pure plunge. For both, the effective angle of attack time history is given by Equation 1 .

$$
\alpha_{e f f}=\alpha_{0}+\lambda \arctan (\pi S t) \cos \{2 \pi(f t+\phi)\}+\arctan \{\pi S t \sin (2 \pi f t)\}
$$

Both sets of kinematics use a Strouhal number equal to 0.08 , a reduced pitching frequency of $k=0.25$, and a mean angle of attack of $8^{\circ}$. For the pitch-plunge motion the plunge component lags the pitch component by a quarter of the period and the ratio of pitch amplitude to plunge induced angle of attack, $\lambda$, is 0.6 . The $\lambda$ parameter governs how much pitch reduces the plunge-induced effective angle of attack. For pure plunge, $\lambda=0$. The effective angle of attack history is plotted in Figure 3.

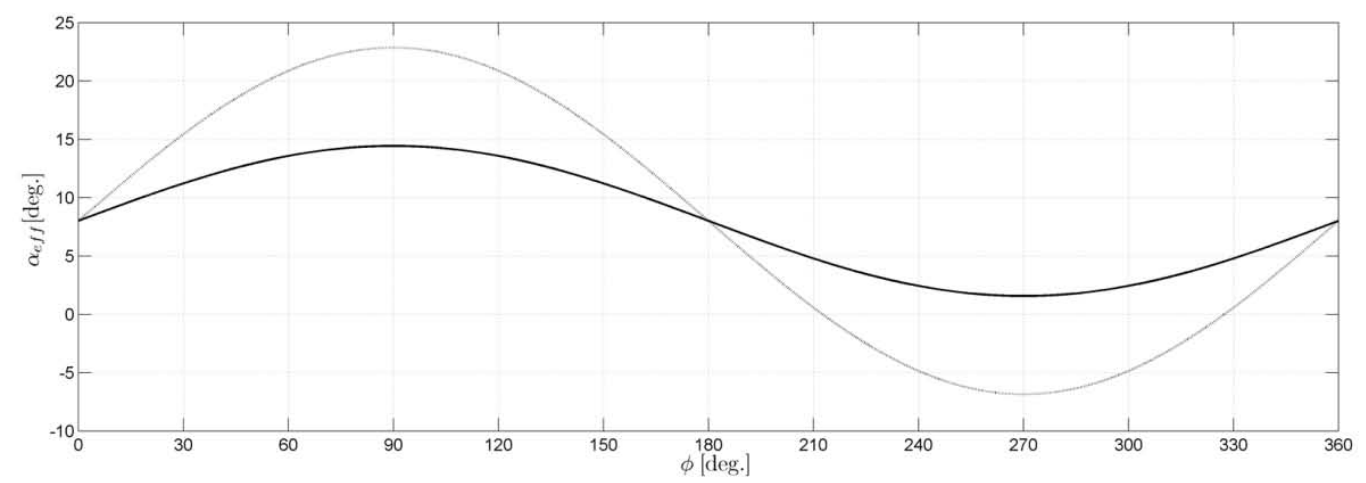

Figure 3. Effective angle of attack time histories for pitch-plunge (solid line) and plunge (dotted line).

The pitch-plunge was motivated by the desire for kinematics that maximize propulsive efficiency ${ }^{5}$. The pitchplunge kine matics were designed for a SD7003 airfoil and the effective angle of attack would make s mall excursions in effective angle of attack past the SD7003's static stall angle of attack. Therefore, for the SD7003 the pitch-plunge kine matics would result in relatively weak dynamic stall. The pure-plunge was simply an attempt to explore deep dynamic stall. Both have been examined in detail for 2D models ${ }^{4,6}$. Ol, et al. ${ }^{2}$ considered the SD7003 airfoil in combined experiment and computation at $\mathrm{Re}=60 \mathrm{~K}$, comparing lift history with Theodorsen's formula for attached flow and planar wakes. The pitch-plunge evinced a trailing edge separation throughout the motion's downstroke and the plunge kinematics resulted in a leading edge separation and a pronounced leading and trailing edge vortex. Kang, et al..$^{6}$ generalized these results to the Re range of $1 \times 10^{4}$ to $6 \times 10^{4}$, and Baik, et al. ${ }^{4}$ compared the SD7003 airfoil to a 2D, finding that the plate produced stronger leading-edge separation but less Reynolds number dependency.

The present study uses one of the previously studied Reynolds numbers (relying on the Re-independence found in Baik, et $a l^{4}$ ) and both sets of motion kinematics. An additional 2D flat plate experiment was performed at the same Re as the 3D flat plate in pure-plunge.

Table 2 lists test conditions and motion phases at which PIV snapshots were taken for the 2D plate, and Table 2 does the same for the $3 \mathrm{D}$ plate. 
Table 2. The 2D flat plate dye flow and PIV test matrix for the five sets of Reynolds number and kinematics

\begin{tabular}{|c|c|c|c|c|}
\hline Experiment ID & Re & Kinematics & $\boldsymbol{\phi}$ & $\begin{array}{c}\text { Realizations per } \\
\text { Phase }\end{array}$ \\
\hline $\mathbf{1}^{\mathbf{4}}$ & 10,000 & Pitch-Plunge & $0^{\circ}, 90^{\circ}, 120^{\circ}, 150^{\circ}, 180^{\circ}, 270^{\circ}$ & 204 \\
\hline $\mathbf{2}^{\mathbf{4}}$ & 30,000 & Pitch-Plunge & $0^{\circ}, 90^{\circ}, 120^{\circ}, 150^{\circ}, 180^{\circ}, 270^{\circ}$ & 204 \\
\hline $\mathbf{3}^{*}$ & 30,000 & Plunge & $0^{\circ}, 90^{\circ}, 120^{\circ}, 150^{\circ}, 180^{\circ}, 270^{\circ}$ & 200 \\
\hline $\mathbf{4}^{\mathbf{4}}$ & 60,000 & Pitch-Plunge & $0^{\circ}, 90^{\circ}, 120^{\circ}, 150^{\circ}, 180^{\circ}, 270^{\circ}$ & 200 \\
\hline $\mathbf{5}^{\mathbf{4}}$ & 60,000 & Plunge & $0^{\circ}, 90^{\circ}, 120^{\circ}, 150^{\circ}, 180^{\circ}, 270^{\circ}$ & 200 \\
\hline
\end{tabular}

Two-dimensional flat plate experiment not performed in Baik, et al ${ }^{4}$.

Table 3. The 3D flat plate dye flow and DPIV test matrix

\begin{tabular}{|c|c|c|c|c|}
\hline Experiment ID & Re & Kinematics & $\boldsymbol{c}$ & $\begin{array}{c}\text { Realizations per } \\
\text { Phase }\end{array}$ \\
\hline $\mathbf{6}$ & 30,000 & Pitch-Plunge & $0^{\circ}, 90^{\circ}, 120^{\circ}, 150^{\circ}, 180^{\circ}, 270^{\circ}$ & 200 \\
\hline 7 & 30,000 & Plunge & $0^{\circ}, 90^{\circ}, 120^{\circ}, 150^{\circ}, 180^{\circ}, 270^{\circ}$ & 200 \\
\hline
\end{tabular}

Results for $\phi=270^{\circ}$ (halfway through the upstroke; effective angle of attack $\left(\alpha_{\text {eff }}\right)_{\phi=270^{\circ}}=1.6^{\circ}$ for pitchplunge and $\left(\alpha_{\mathrm{eff}}\right)_{\phi=270^{\circ}}=-6.85^{\circ}$ for plunge) is deemphasized, as the flow is mostly attached.

\section{A. Two-Dimensional Model: Pitching and Plunging Motion (Baik, et al ${ }^{4}$.)}

$2 \mathrm{D}$ pitch-plunge at $\mathrm{Re}=30,000$ was presented in Baik, et at . and is reproduced here to compare with the 3D plate. The flow visualization and PIV data is presented in Figure 4 and Figure 5, respectively. Figure 4 presents a comparis on of flow visualization, $\bar{u}$, and $\omega^{*}$ for the pitching and plunging flat plate. Figure 5 presents the phaseaveraged second-order turbulence statistics.

The pitching and plunging two-dimensional model begins the motion at $\phi=0^{\circ}$ with transitional vorticalstructures that are phase-locked near the leading edge. From approximately the half-chord location to the trailing edge the flow is attached. From $\phi=90^{\circ}-180^{\circ}$ a region of growing closed separation forms over the entire chord. The region of closed separation is bounded by a turbulent shear layer that has negative $\vec{u} \vec{v} / U_{\infty}^{2}$. Anisotropy is observed in the velocity correlations with $\overline{u^{\prime 2}}$ more prevalent than $\overline{v^{\prime 2}}$. 


\begin{tabular}{ccccccc}
\multicolumn{1}{c}{$\omega^{*}$} \\
\hline${ }_{-30}$ & -20 & -10 & 0 & 10 & 20 & 30
\end{tabular}

$0^{\circ}$
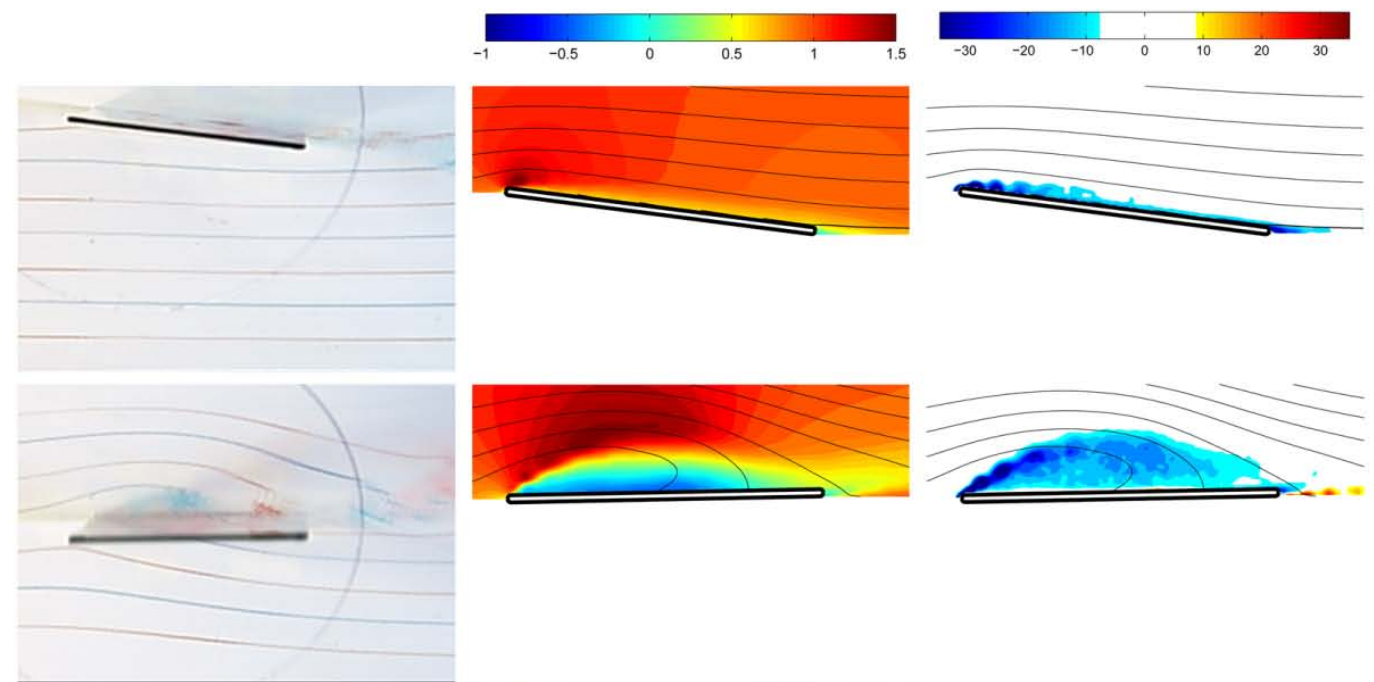

$90^{\circ}$
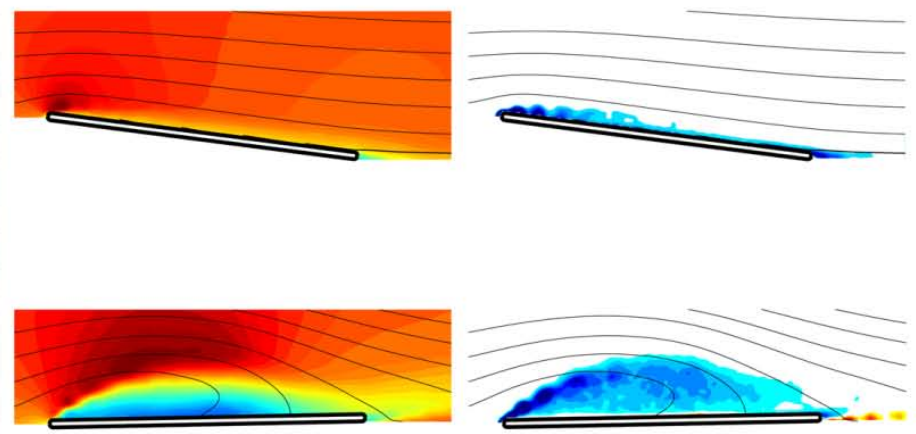

$120^{\circ}$
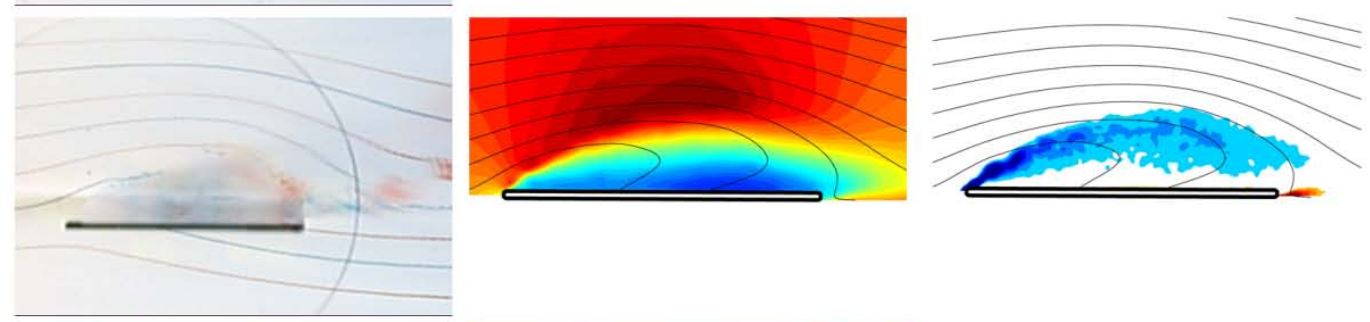

$150^{\circ}$
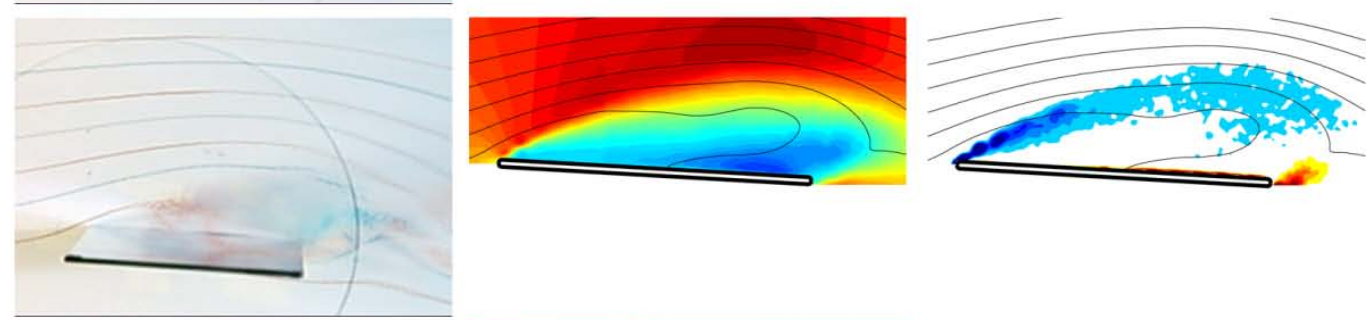

$180^{\circ}$
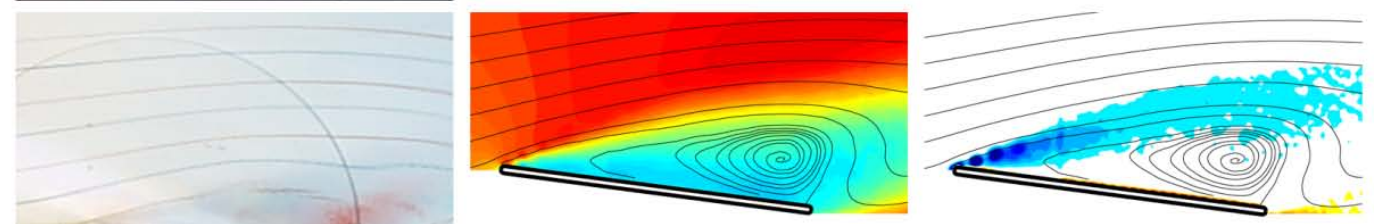

$270^{\circ}$
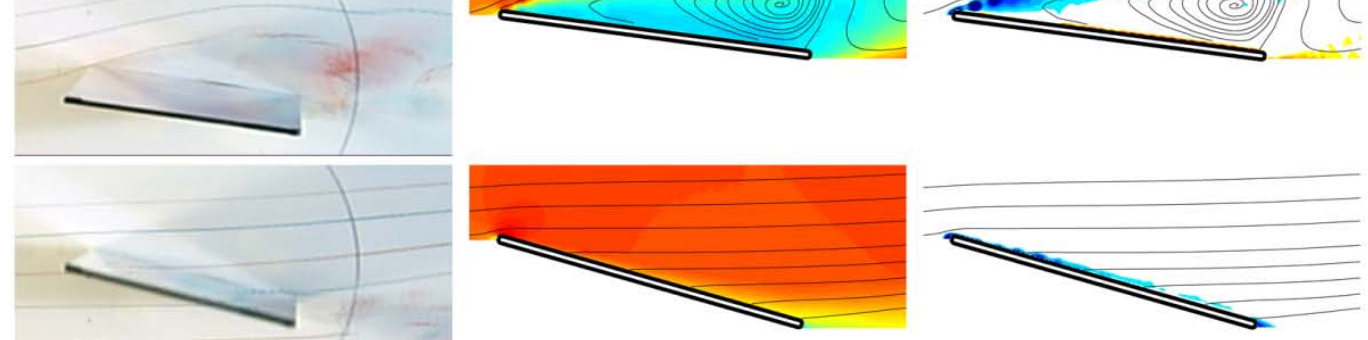

Figure 4. Flow visualization, $\bar{u}$, and $\omega^{*}$ for the pitching and plunging $2 D$ flat plate at $R e=30,000$ 


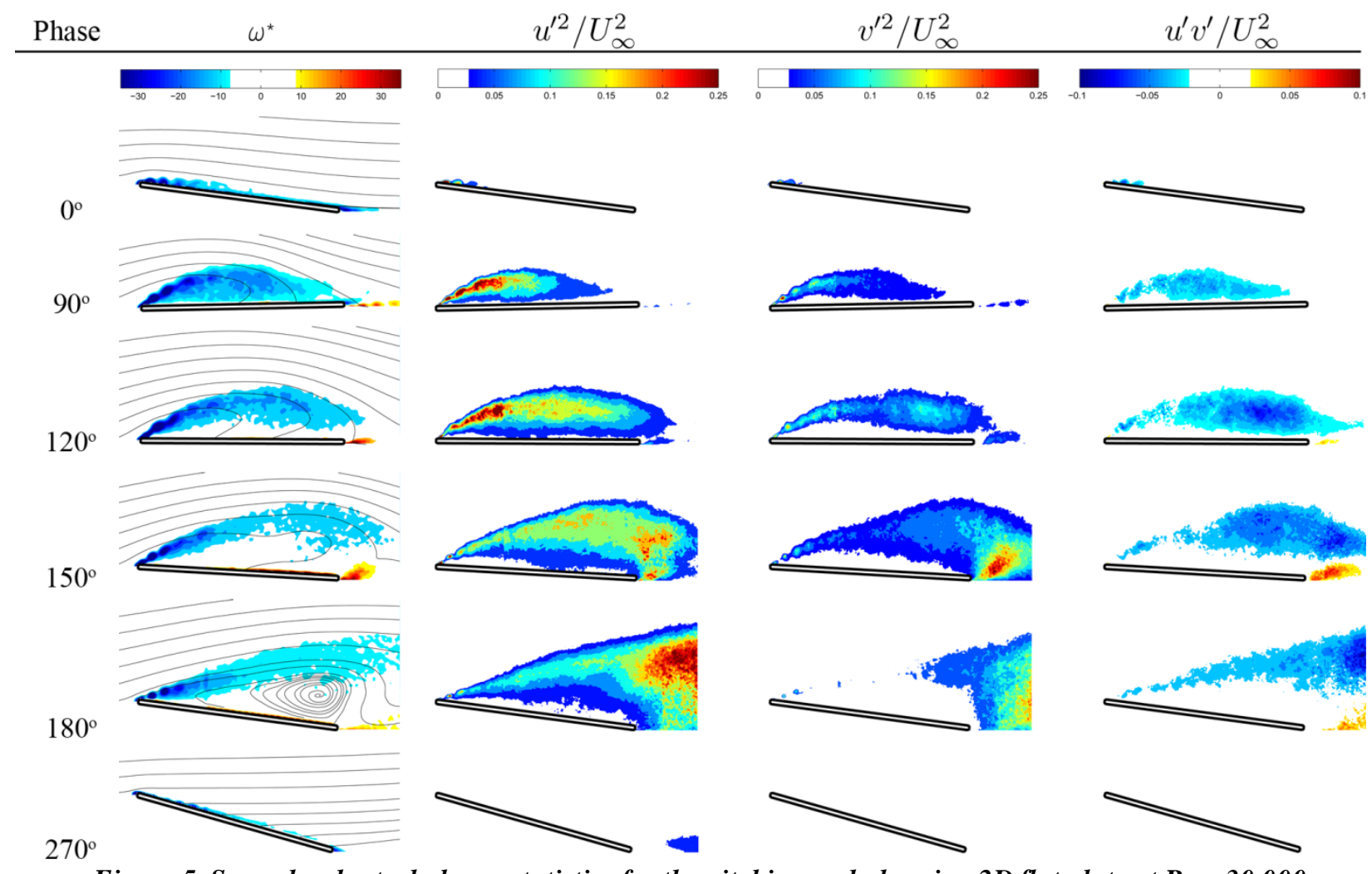

Figure 5. Second order turbulence statistics for the pitching and plunging $2 D$ flat plate at $R e=30,000$

\section{B. Two-Dimensional Model: Plunging Motion}

Pure-plunge 2D flat plate data are shown in Figure 6 (dye flow visualization, $\bar{u}$, and $\omega^{*}$ ) and Figure 7 ( vorticity and second-order phase-averaged turbulence statistics).

For the plunging 2D flat plate the dramatic changes in flow topology can be attributed to the large effective angle of attack. The dominant characteristic of the flowfield is the formation of large leading and trailing edge vortices. At $\phi=0^{\circ}$ the vorticity field shows a small region of leading edge separation with phase-locked transitional vorticalstructures (confirmed by the turbulence statistics in Figure 7). The leading edge separation then grows to a large leading edge vortex by $\phi=90^{\circ}$. The leading edge vortex has negative $\overrightarrow{u^{\prime} v} / U_{\infty}^{2}$ values along with $\overline{u^{\prime 2}} / U_{\infty}^{2}$ and $\overrightarrow{v^{\prime 2}} /$ $U_{\infty}^{2}$ of equal magnitude. The turbulence statistics suggest that the turbulent flow in the leading edge vortex is isotropic. For the leading edge vortex, turbulence statistics associated with phase-locked vortical-structures can be the result of cycle-to-cycle variation in the location of vortices. The leading edge vortex convects downstream where it interacts with the trailing edge vortex at $\phi=120^{\circ}$. The turbulence statistics are isotropic with negative and positive $\vec{u} \vec{v} / U_{\infty}^{2}$. The negative $\overrightarrow{u^{\prime} v} / U_{\infty}^{2}$ is associated with the shed leading edge vortex and a reg ion of positive vorticity created by the interaction of the leading edge vortex with surface of the flat plate. The positive $\vec{u} \vec{v} / U_{\infty}^{2}$ is associated with the growing trailing edge vortex. By $\phi=150^{\circ}$ the leading edge vortex has left the PIV field-ofview and the trailing edge vortex is convecting downstream. At $\phi=180^{\circ}$ the leading and trailing edge vortices have left the PIV field-of-view and one finds open separation. The turbulence statistics of the last two phases of the downstroke evince isotropic turbulence in the leading edge shear layer that bounds the flow separation. 


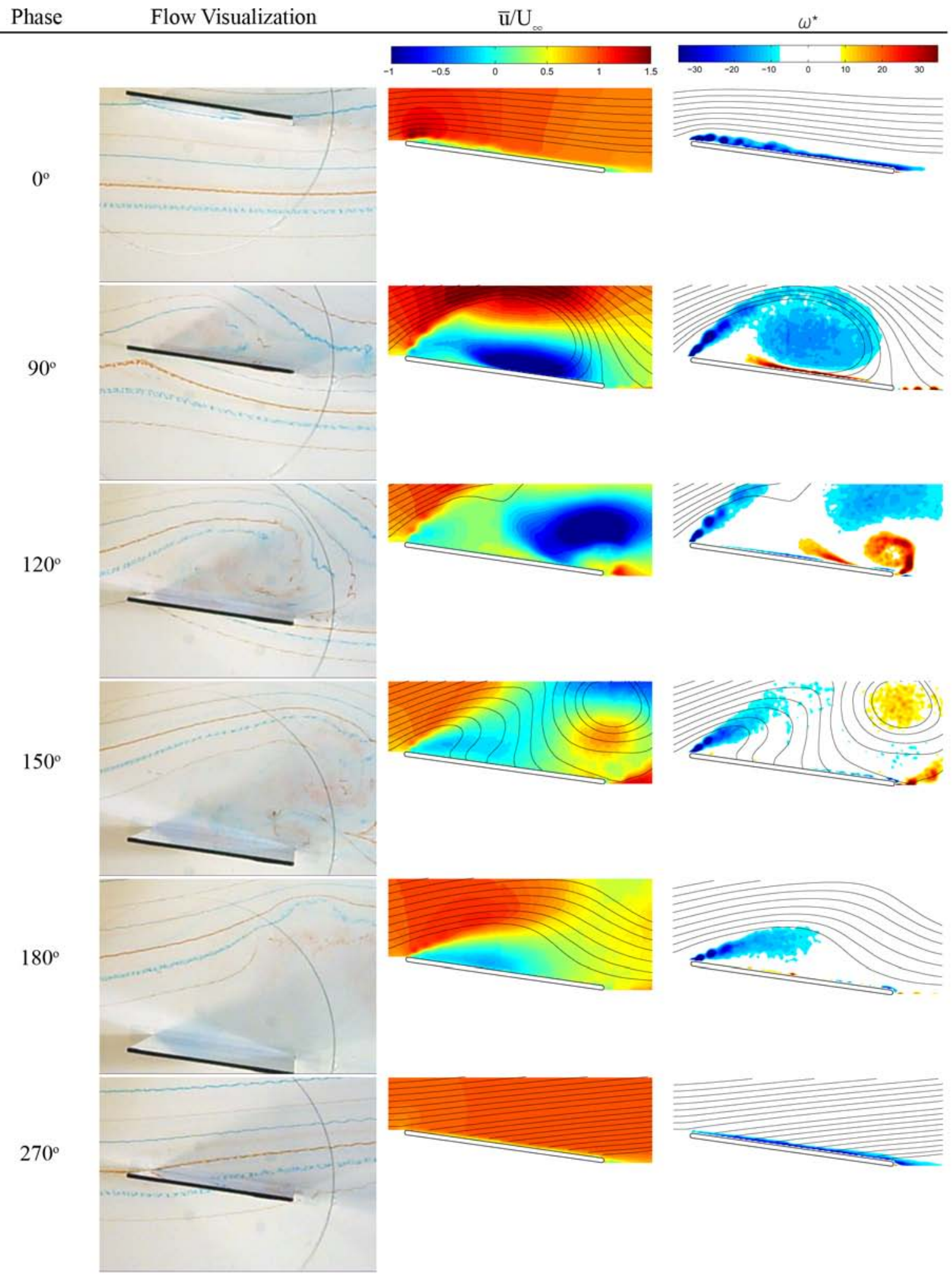

Figure 6. Flow visualization, $\bar{u}$, and $\omega^{*}$ for the plunging two-dimensional flat plate at $R e=30,000$ 


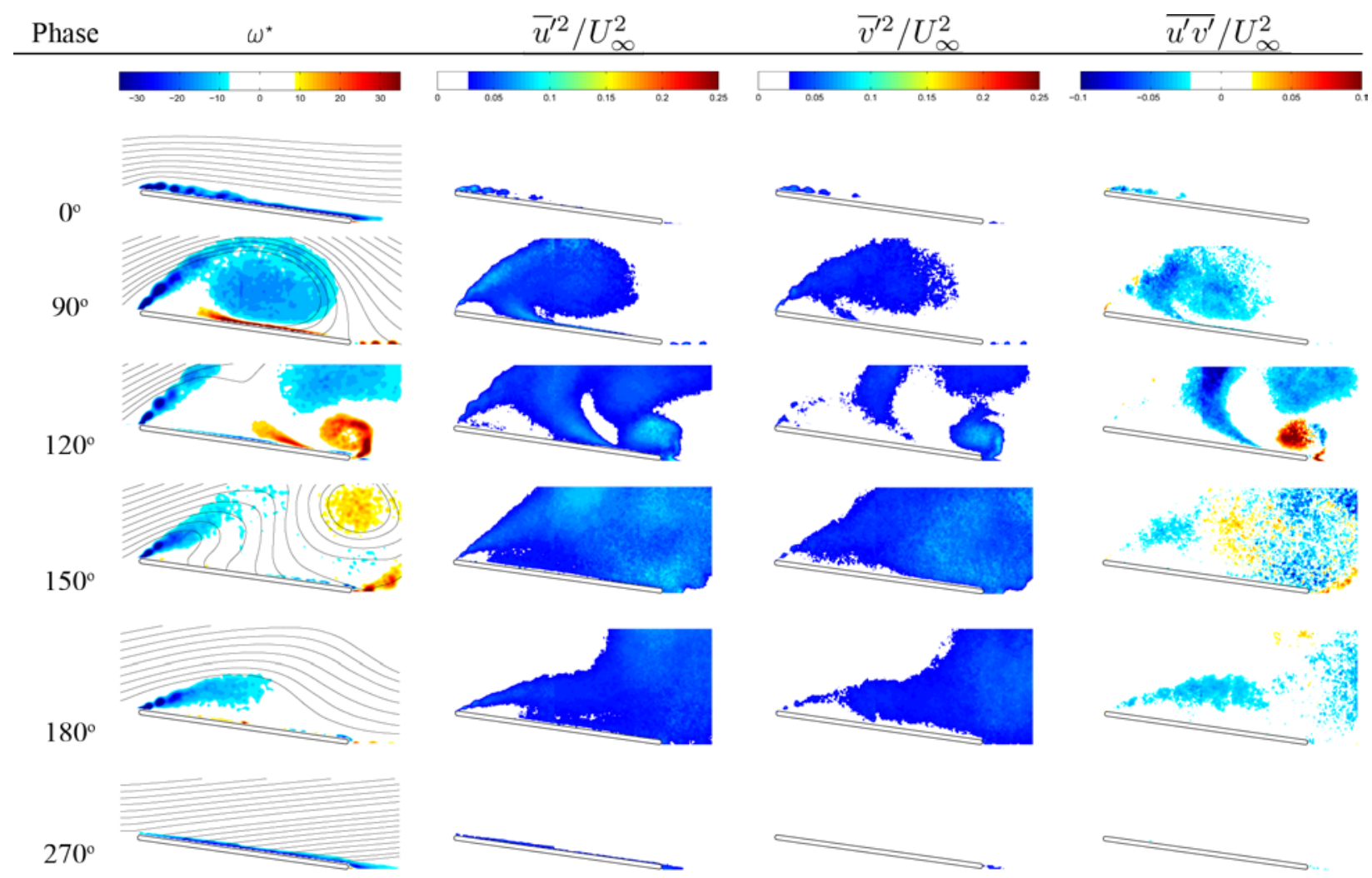

Figure 7. Second order turbulence statistics for the plunging $2 \mathrm{D}$ flat plate at $\mathrm{Re}=30,000$

\section{Three-Dimensional Model: Pitching and Plunging Motion}

Pitching-plunging 3D plate results are given in Figure 8 (dye flow visualization, $\bar{u}$, and $\omega^{*}$ ) and Figure 9 (vorticity with the second order turbulence statistics) for the plunging flat plate. Figure 9 compares the.

Similar to the 2D model results at $\phi=0^{\circ}$, phase-locked transitional vortical-structures are observed near the leading edge. These promote reattachment. The most notable observation of the pitching and plunging 3D flat plate is the region of closed separation that occurs from $\phi=90^{\circ}-150^{\circ}$. In Figure 8 at $\phi=90^{\circ}$ the location and size of this closed leading edge separation do not significantly change as the flow temporally evolves. Independent of model motion or Reynolds number, for the 3D flat plate a region of closed leading edge separation is present at $\phi=90^{\circ}$ that affects the flow over the entire chord as the flow reattachment point moves toward the trailing edge. The growth of the closed leading edge separation is not present for the three-dimensional flat plate. Examination of the streamlines in Figure 8 shows a region of closed separation with the same approximate spatial extent from $\phi=90^{\circ}-150^{\circ}$. Examination of the turbulence statistics during these phases yields negative $u^{\prime} v^{\prime} / U_{\infty}^{2}$ for the closed separation's bounding shear layer. 


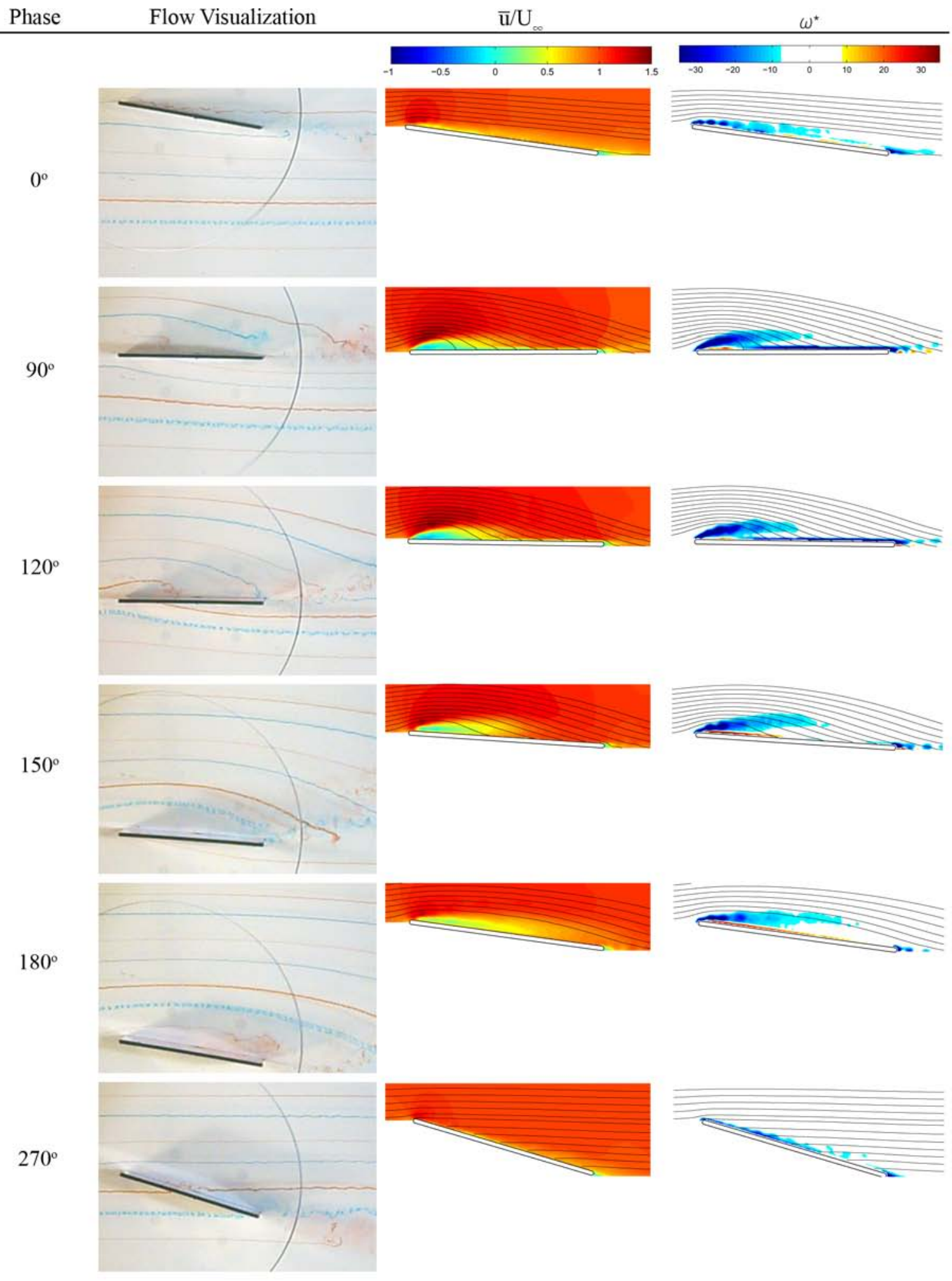

Figure 8. Flow visualization, $\bar{u}$, and $\omega^{*}$ for the pitching and plunging $3 D$ flat plate at $R e=30,000$ 


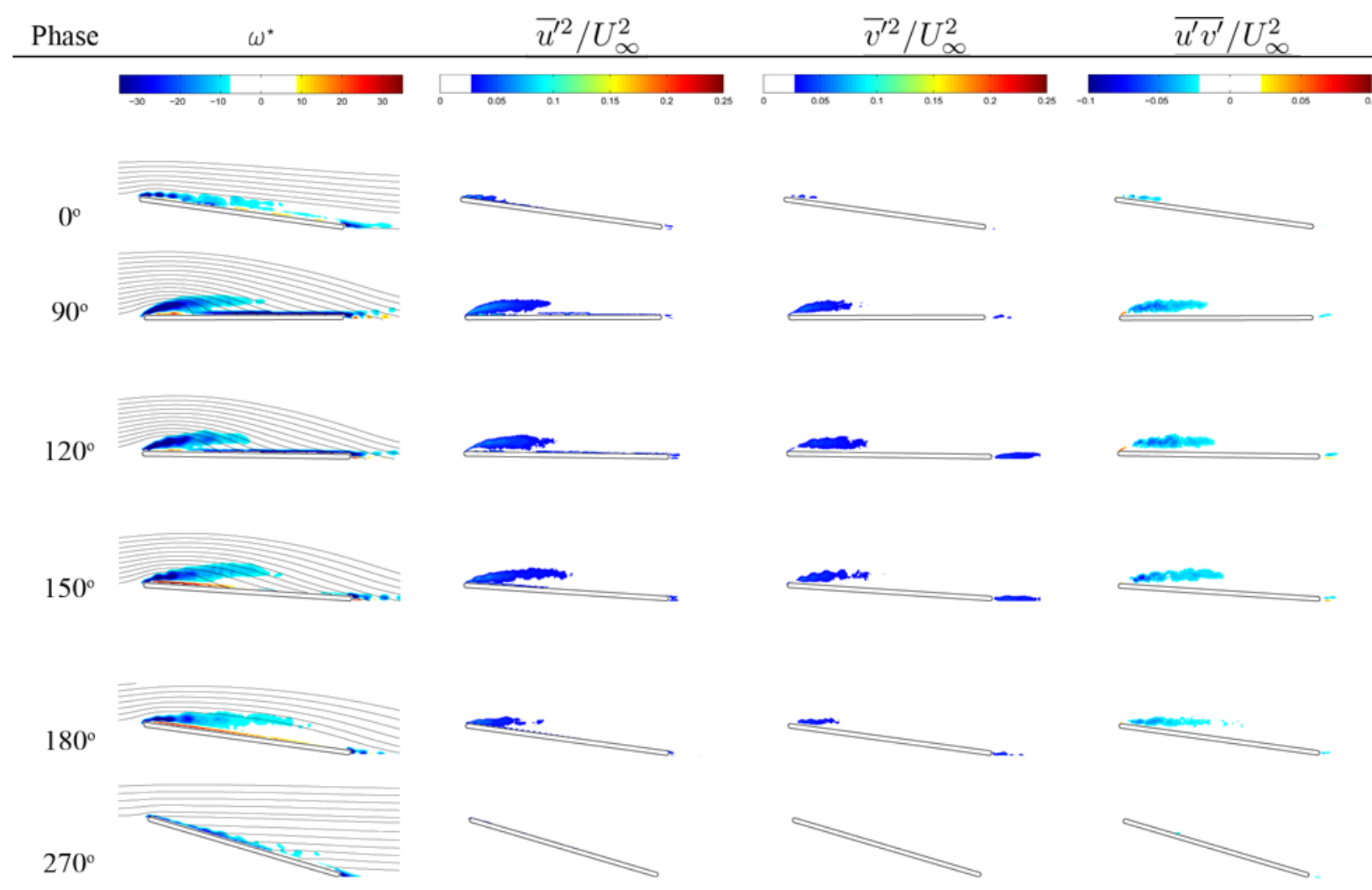

Figure 9. Turbulence statistics for the pitching and plunging 3D plate at Reynolds number 30,000.

\section{Three-Dimensional Model: Plunging Motion}

Pure-plunge 3D flat plate results are presented in Figure 10 (dye flow visualization, $\bar{u}$, and $\omega^{*}$ ) and Figure 11 (vorticity with the second order turbulence statistics).

Again, at $\phi=0^{\circ}$ phase-locked transitional vortical-structures are observed near the leading edge that promote reattachment. Similar to the pitching and plunging 3D flat plate, the most notable observation of the plunging 3D flat plate is the region of closed separation that occurs from $\phi=90^{\circ}-150^{\circ}$. The dynamics of the closed separation region are interesting when compared to the large leading and trailing edge vortices generated under the same experimental conditions for the 2D flat plate. In Figure 10 the location and size of this closed separation at $\phi=90^{\circ}$ does not significantly change as the flow temporally evolves. Examining the $2 \mathrm{D}$ flat plate under the same experimental conditions at $\phi=90^{\circ}$ yields the observation of a large leading edge vortex. At $\phi=90^{\circ}$ the large leading edge vortex affects the flow over the entire chord with the attaching near the trailing edge. The separation for the 2D flat plate is in stark contrast with the 3D flat plate. Examination of the streamlines shows a region of closed separation with the same approximate spatial extent from $\phi=90^{\circ}-150^{\circ}$. Examination of the turbulence statistics during these phases yields negative $u^{\prime} v^{\prime} / U_{\infty}^{2}$ for the closed separation's bounding shear layer. 


\begin{tabular}{ccccccc}
\multicolumn{10}{c}{$\omega^{*}$} & & & \\
\hline & & & & & & \\
\hline-30 & -20 & -10 & 0 & 10 & 20 & 30
\end{tabular}
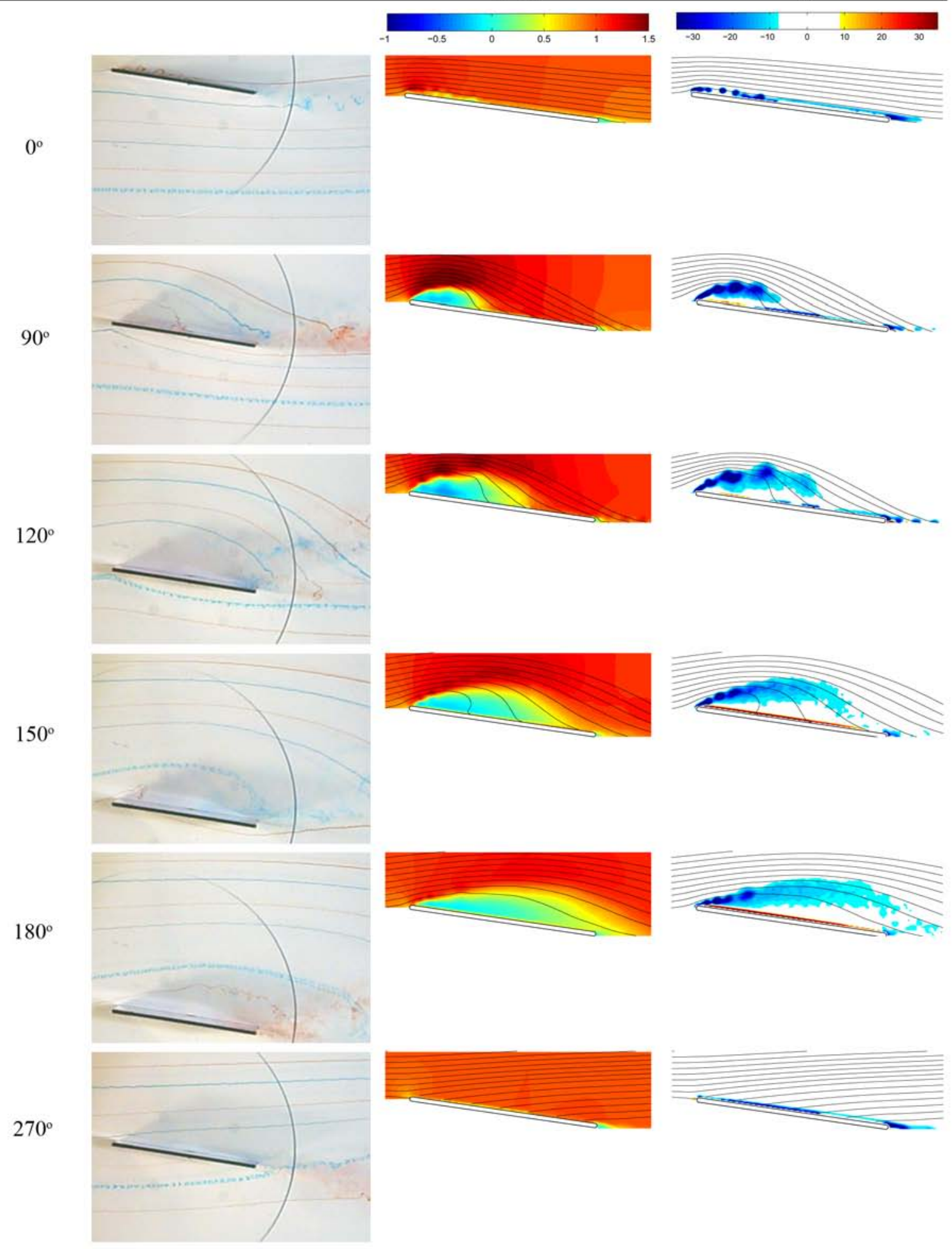

Figure 10. Flow visualization, $\bar{u}$, and $\omega^{*}$ for the plunging $3 D$ flat plate at $R e=30,000$ 


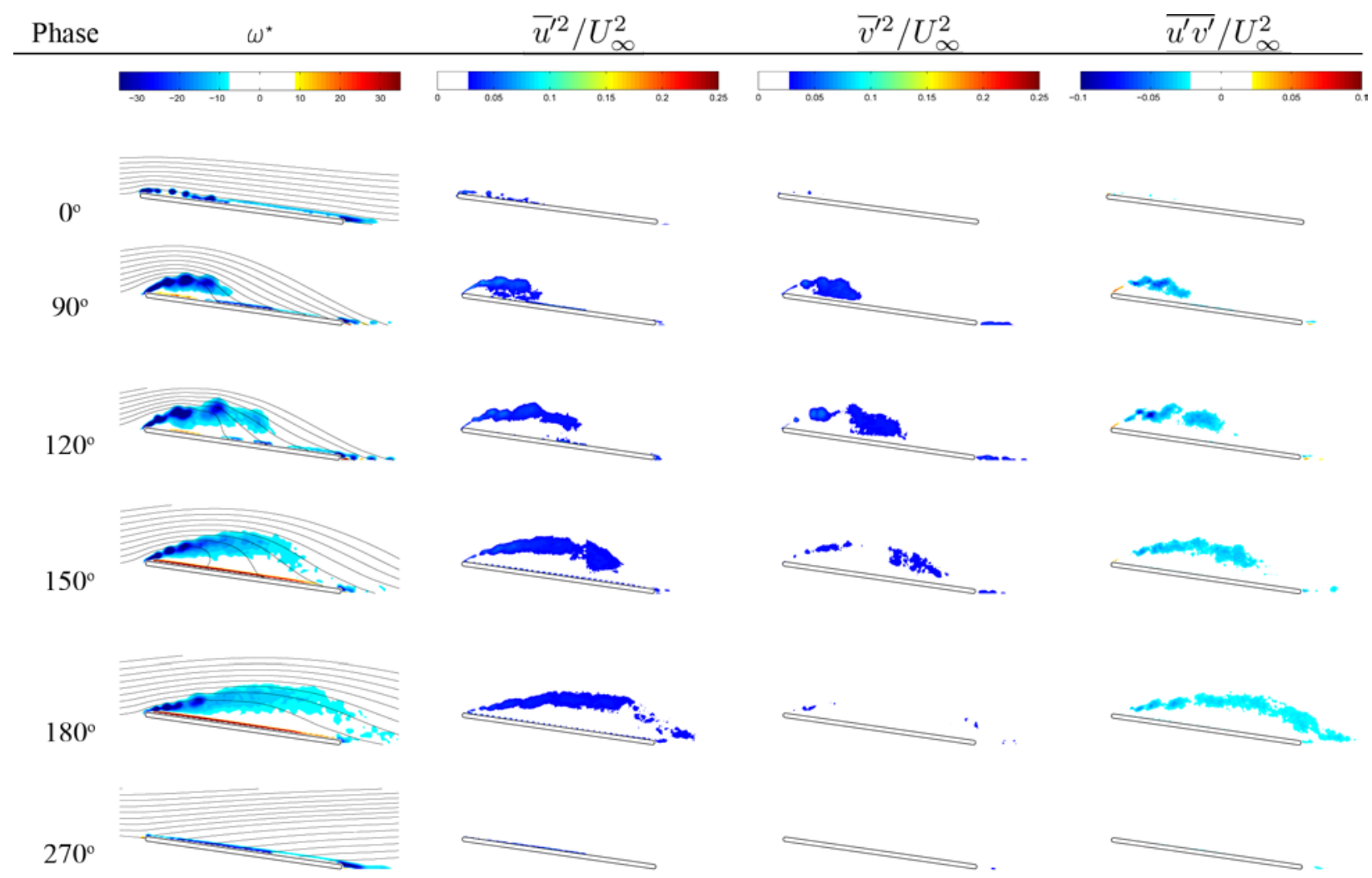

Figure 11. Turbulence statistics for the pure plunging $3 D$ plate at $R e=30,000$.

\section{E. Three-Dimensional Model: Uni versity of Michigan and AFRL Comparison}

Using the same kinematic parameters as the 3D flat plate for the UM PIV at Reynolds number 30,000, experimental validation was provided by the AFRL HFWT using an AR $=2$ flat plate at the same Reynolds number. The normalized $\overline{\mathrm{u}}$ and vorticity fields comparing the AFRL and UM experiments are plotted in Figure 12 and Figure 13 , the former figure compares pitching and plunging while the latter figure compares plunging.

The contours of normalized $\overline{\mathrm{u}}$ and vorticity in Figure 12 show qualitative agreement between the AFRL and UM with the main discrepancy being the chordwise extent of the flow separation. As previously observed in the UM PIV the separation that occurs at the leading edge is closed and the majority of the flow over the chord is attached through the downstroke. The AFRL separation at $\phi=90^{\circ}-180^{\circ}$ behaves similarly except that the separation consistently occupies larger fractions of the chord.

At $\phi=0^{\circ}$ both pitching and plunging PIV data sets have vortical structures in the leading edge shear layer. At subsequent phases the vortical instabilities are only present in the UM PIV.

The contours of normalized $\overline{\mathrm{u}}$ and vorticity in Figure 13 shows qualitative agreement between the AFRL and UM. Again the main discrepancy between the data sets is the chordwise extent of the flow separation. The separation through $\phi=90^{\circ}-180^{\circ}$ is comparable but the AFRL separation consistently occupies larger fractions of the chord.

At all phases of the downstroke the UM plunging PIV data has vortical structures in the leading edge shear layer. The AFRL plunging PIV never has this property throughout the motion. 


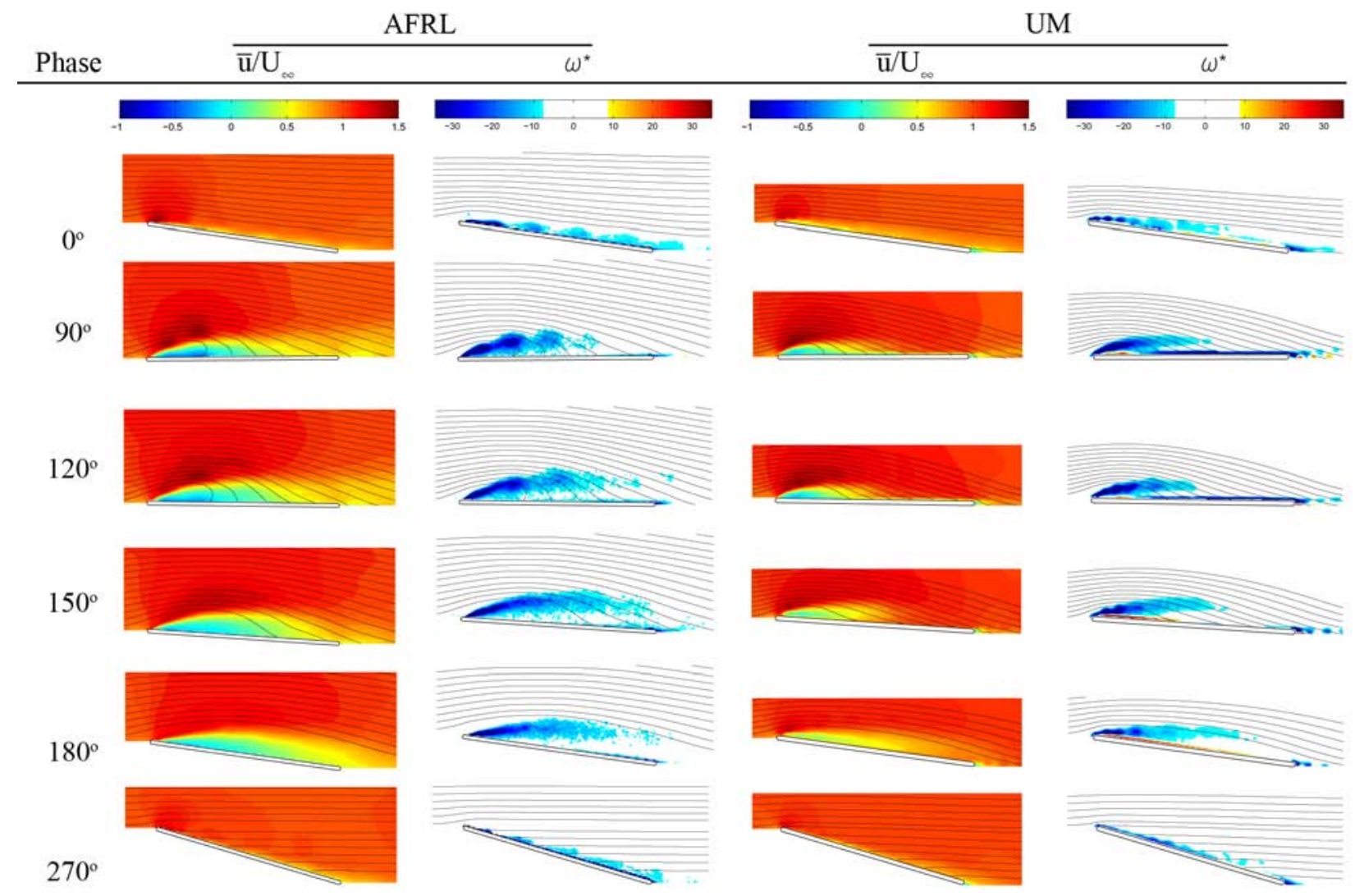

Figure 12. $\bar{u}$ and $\omega^{*}$ for the pitching and plunging $3 D$ flat plate at $R e=30,000$ (AFRL/UM comparison) 


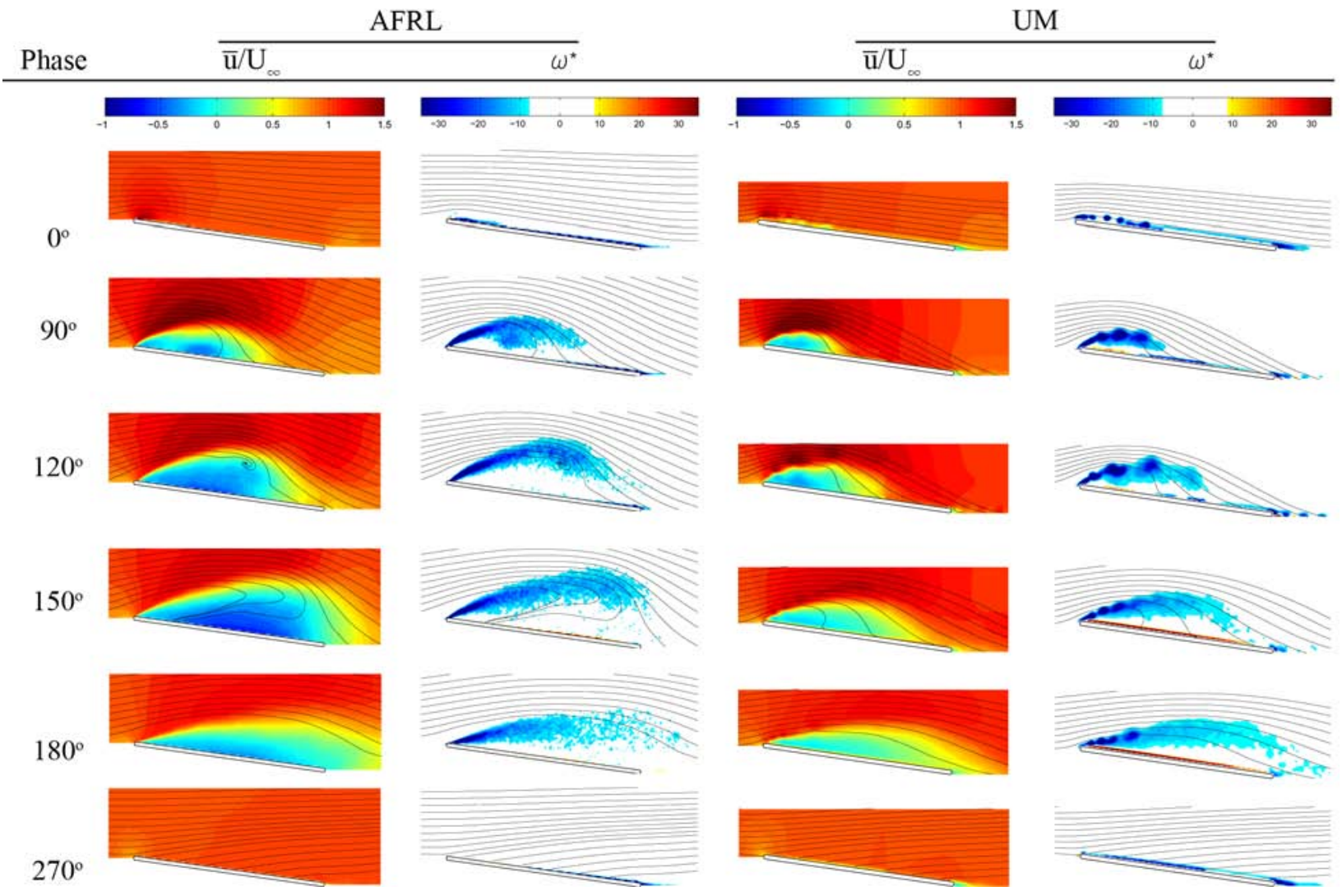

Figure 13. $\bar{u}$ and $\omega^{*}$ for the plunging 3 D flat plate at Re $=30,000$ (AFRL/UM comparison)

Distributions of normalized $\overline{\mathrm{u}}$ were plotted at chordwise locations to quantify the difference between the AFRL and UM PIV. Since the qualitative differences between data sets were observed in Figure 12 and Figure 13 from $\phi=90^{\circ}-180^{\circ}$, these phases were used to plot distributions of normalized $\overline{\mathrm{u}}$ in Figure 14 (pitch-plunge comparis on) and Figure 15 (plunge comparison).

For the comparis on of the pitching and plunging 3D flat plates in Figure 14 the AFRL separation affects a larger portion of the chord than the UM separation. This effect is best illustrated at the 50 and $75 \%$ chord locations where the AFRL normalized $\overline{\mathrm{u}}$ distribution is consistently more negative than the UM. However, the AFRL and UM data both have closed separation through the downstroke.

For the comparis on of the plunging 3D flat plate in Figure 15 the AFRL separation has much stronger effect on the normalized $\overline{\mathrm{u}}$ distribution and it consistently occupies larger fractions of the chord. Both data sets have closed separation through $\phi=90^{\circ}-150^{\circ}$. At $\phi=180^{\circ}$ the AFRL data evinces open separation while the UM data evinces closed separation. 

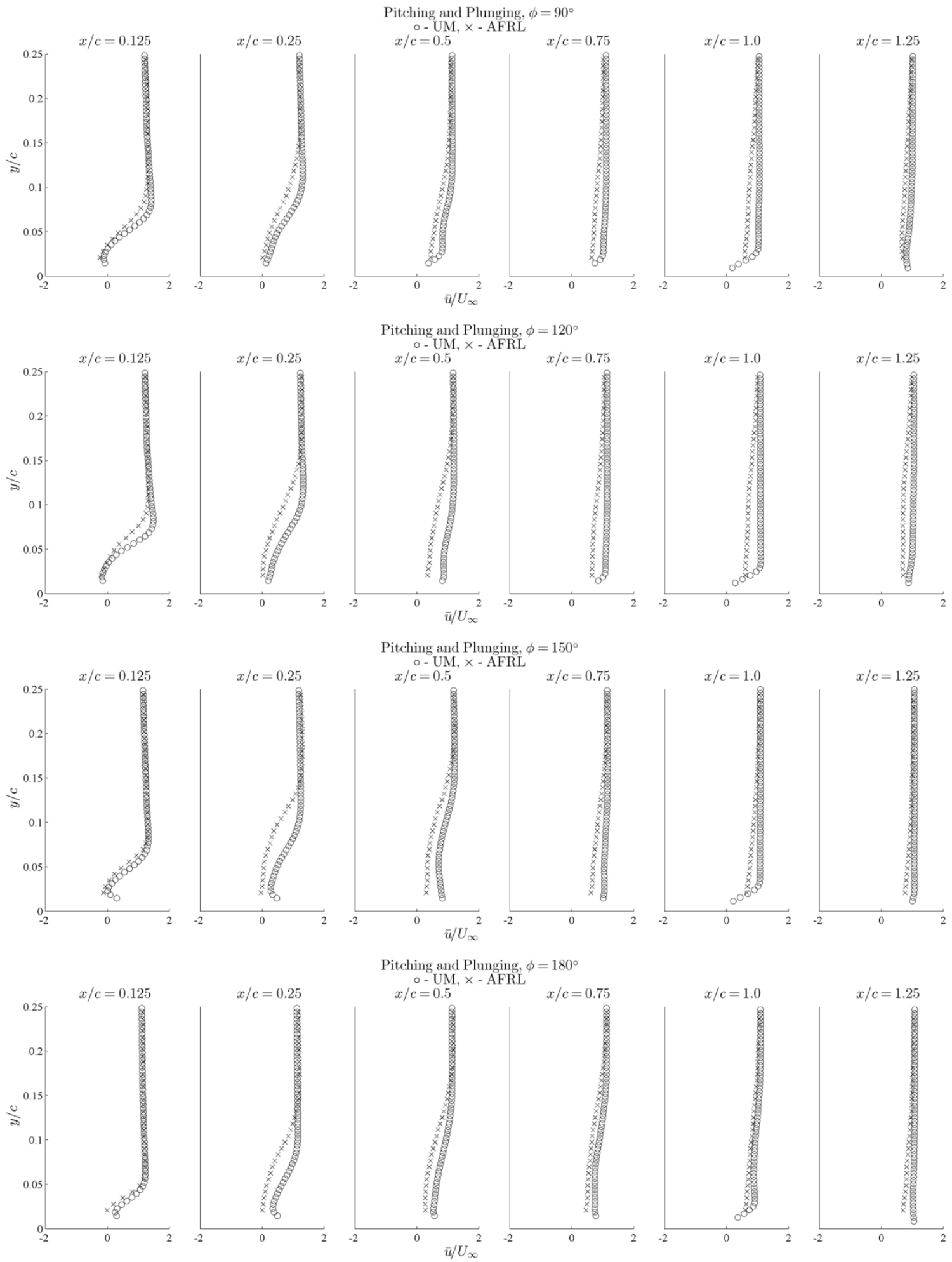

Figure 14. University of Michigan and AFRL pitching and plunging three-dimensional flat plates at $\phi=90^{\circ}-$ $180^{\circ}$, distributions of normalized $\bar{u}$ in the $y$-direction at selected chordwise stations $(x / c=0$ corresponds to the leading edge) 

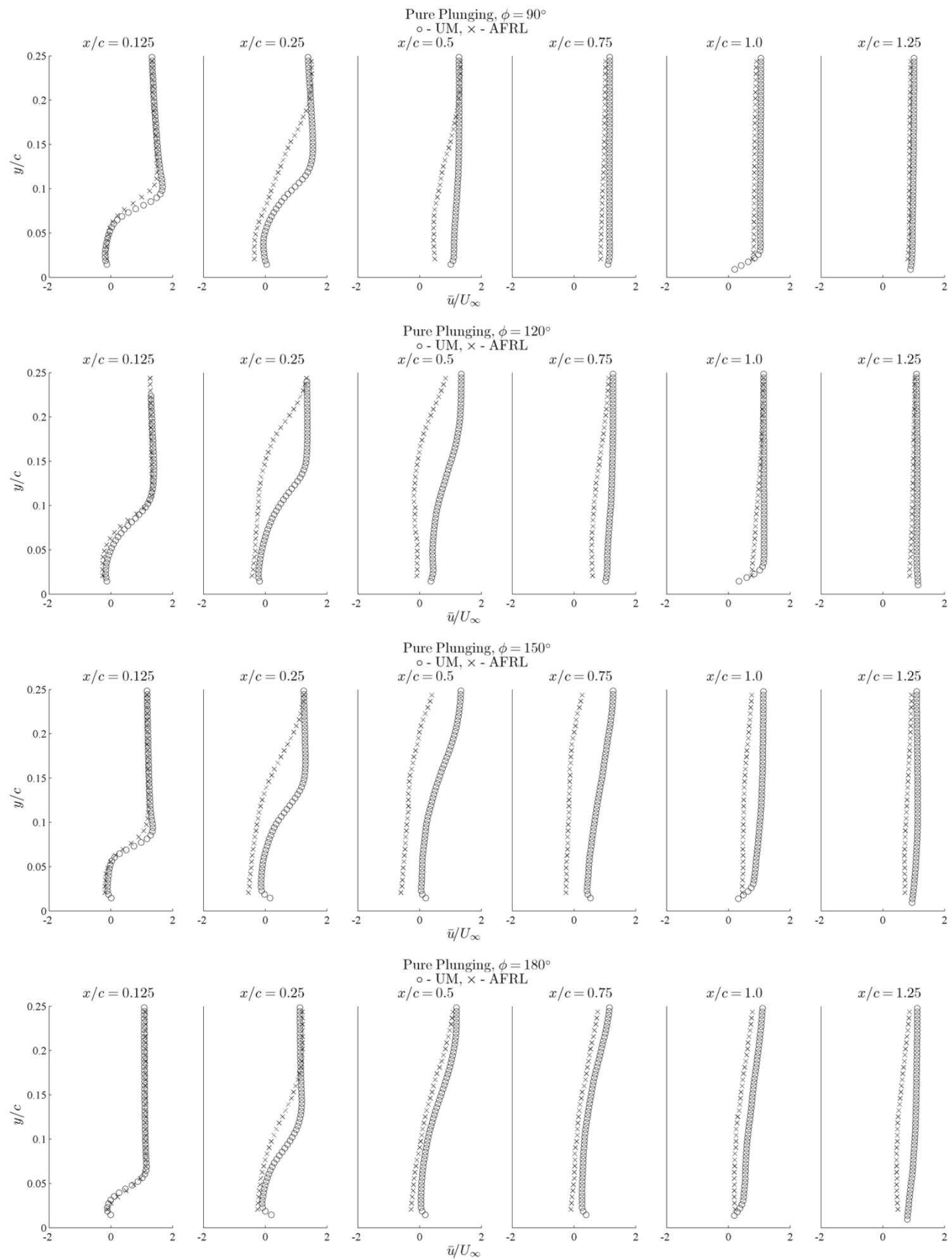

Figure 15. University of Michigan and AFRL plunging three-dimensional flat plates at $\phi=90^{\circ}-1^{\circ}$, distributions of normalized $\bar{u}$ in the $y$-direction at selected chordwise stations $(x / c=0$ corresponds to the leading edge) 


\section{Discussion}

\section{A. Re effect on two-dimensional flat plate}

Re-independence was shown in Baik, et $a t^{4}$. for the pitching and plunging 2D flat plate, and similar reasoning suggested Re-independence for pure-plunge as well. Here we check the latter hypothesis .

Comparison of the contours of normalized $\bar{u}$ and $\omega$ in Figure 6 and Figure A.1 (repeated from Baik, et $a l^{4}$ ) provides a qualitative indication of Re dependence. The comparis on of the contours shows a qualitative difference at $\phi=120^{\circ}$ and $150^{\circ}$ in the interaction of the leading and trailing edge vortices. The Reynolds number 30,000 trailing edge vortex forms earlier than the Reynolds number 60,000 trailing edge vortex. For a more in depth analysis of this difference the normalized $\bar{u}$ distributions are plotted in Figure 16.

The normalized $\bar{u}$ distributions in Figure 16 are plotted at $\phi=90^{\circ}-150^{\circ}$. The observations of the normalized $\bar{u}$ and $\omega$ contours is confirmed by the distributions in Figure 16. At $\phi=120^{\circ}$ and $150^{\circ}$ the chord locations near the trailing edge $(x / c=75 \%$ and $100 \%)$ show that the different trailing edge vortex formations have a significant effect on the normalized $\bar{u}$ distribution.
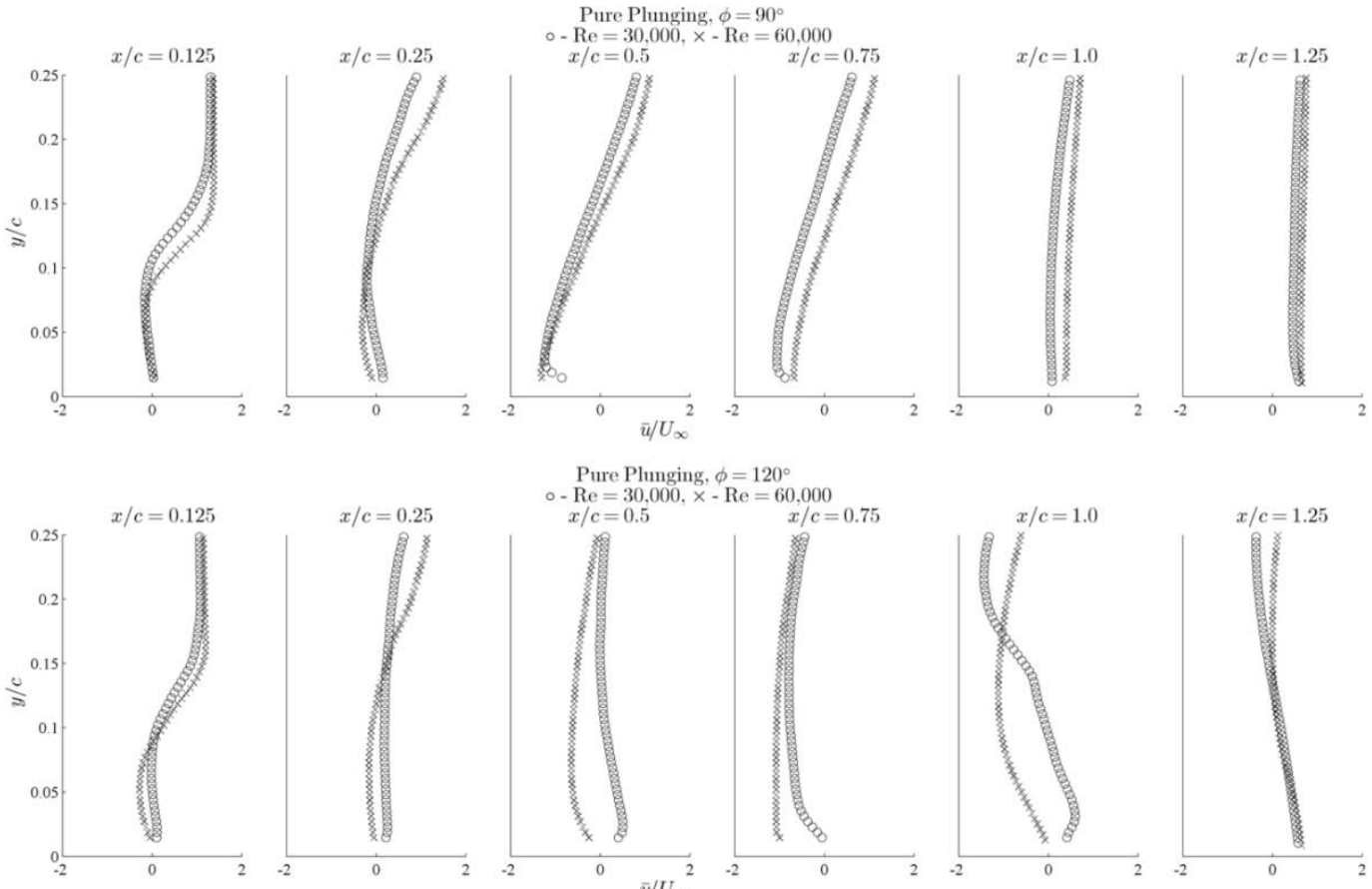

Pure Plunging, $\phi=120^{\circ}$
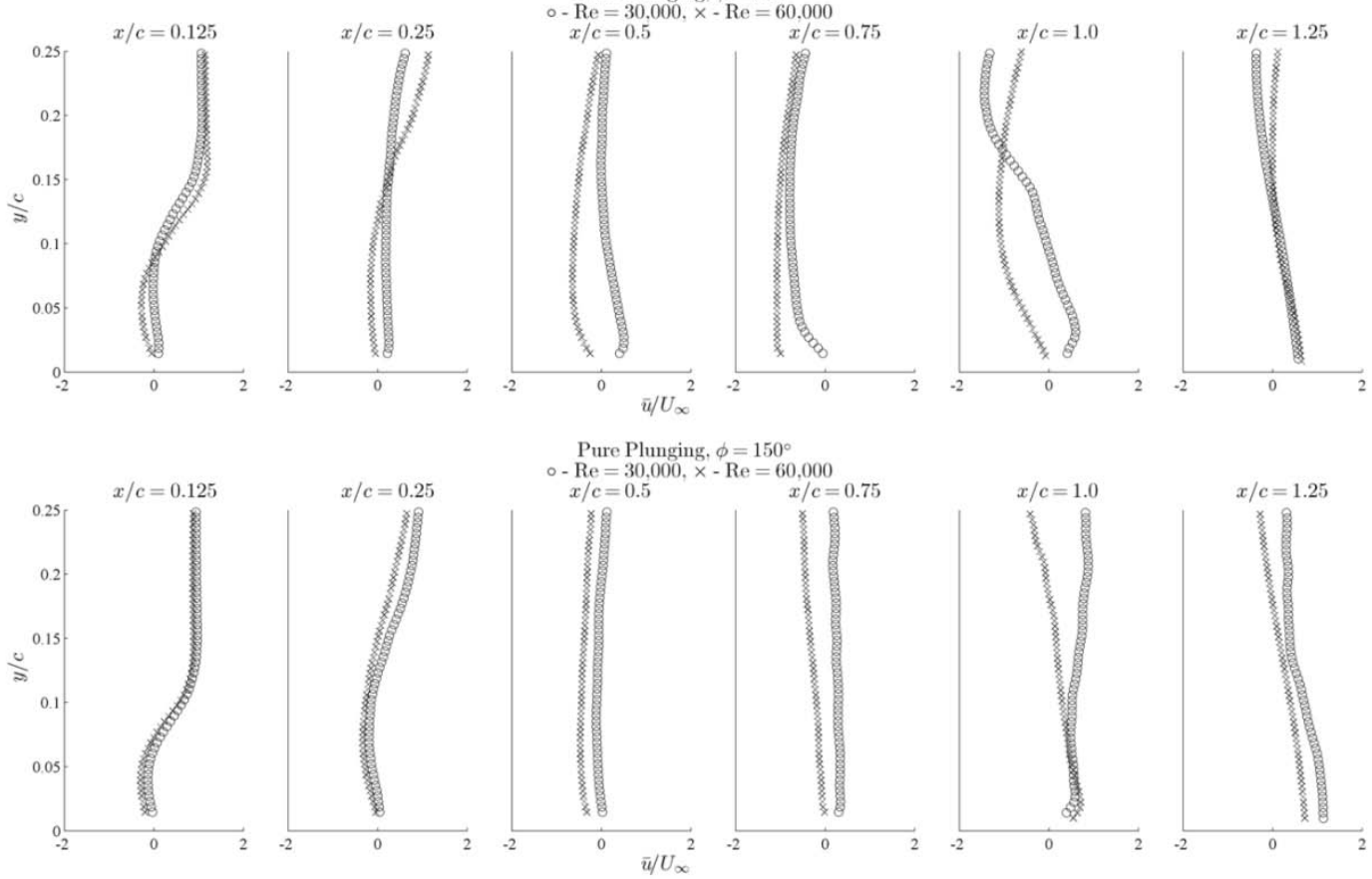

Figure 16. Reynolds number 30,000 and 60,000 for plunging motion at $\phi=90^{\circ}-150^{\circ}$, distributions of normalized $\bar{u}$ in the $y$-direction at selected chordwise stations $(x / c=0$ corresponds to the leading edge $)$ 


\section{B. Aspect ratio effect on flow separation}

The primary objective of the current work is to examine the difference between the $2 \mathrm{D}$ and $3 \mathrm{D}$ plates. In brief, this difference is the temporal evolution of the closed leading edge separation.

For the 2D plate in either motion, a closed leading edge separation grows towards the trailing edge during the downstroke, and . During the closed leading edge separation either forms a leading edge vortex that separates before the bottom of the downstroke, or the separation becomes open when the reattachment point reaches the trailing edge. Though a similar region of closed leading edge separation forms near the leading edge of the 3D plate, the region of closed separation does not significantly grow during the downstroke. Figure 17 illustrates the different temporal evolutions of the flow separation fro $\mathrm{m} \phi=90-150^{\circ}$ for both plates.
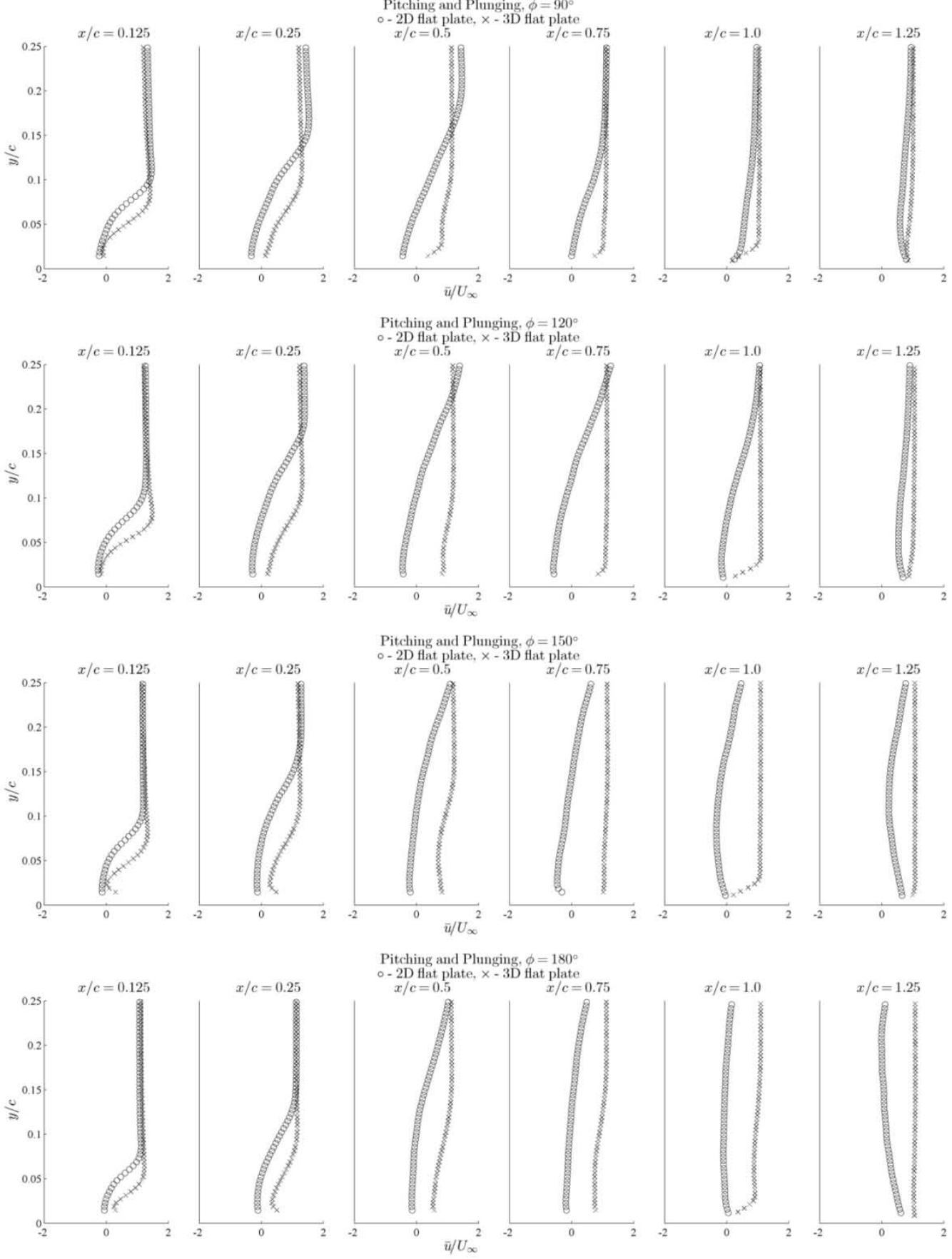

Figure 17. Pitching and plunging two- and three-dimensional flat plates at $\phi=90^{\circ}-180^{\circ}$, distributions of normalized $\bar{u}$ in the $y$-direction at selected chordwise stations $(x / c=0$ corresponds to the leading edge $)$ 
For analyzing the normalized $\bar{u}$ distributions in Figure 17, we focus on the 50\% and $75 \%$ chord locations, since this is the region of the flat plate where the reattachment point is evident.

At $\phi=90^{\circ}$, the difference between the two plates can already be seen at $50 \%$ and $75 \%$ chord. For the 2Dplate the growing separation is affecting these chord locations by reducing normalized $\bar{u}$ and the flow reattaches near the $75 \%$ chord location. The 3D flat plate remains relatively unaffected by comparis on at the same chordwise location and the flow reattaches near the $25 \%$ chord location.

At $\phi=120^{\circ}$ the differences become significant at the $50 \%$ and $75 \%$ chord locations. At the $50 \%$ chord location the difference in normalized $\bar{u}$ near the flat plate's surface for the two- and three-dimensional flat plates is on the order of the freestream velocity. The flow over the two-dimensional flat plate reattaches near the trailing edge and the three-dimensional flat plate, again, reattaches near the $25 \%$ chord location.

At $\phi=150^{\circ}$ the separation region has an even greater influence on the $50 \%$ and $75 \%$ chord locations for the two-dimensional model. The two-dimensional model's normalized $\bar{u}$ distribution not only shows growth of the separated region in the $\mathrm{x}$-direction but also in the y-direction. Similar to the previous two phases of motion the separation of three-dimensional model is limited to influencing the $0 \%$ and $25 \%$ chord locations.

To better quantify the differences between 2D and 3D plate flowfields, Figure 18 compares normalized $\bar{u}$ distributions at $\phi=90^{\circ}-180^{\circ}$. 

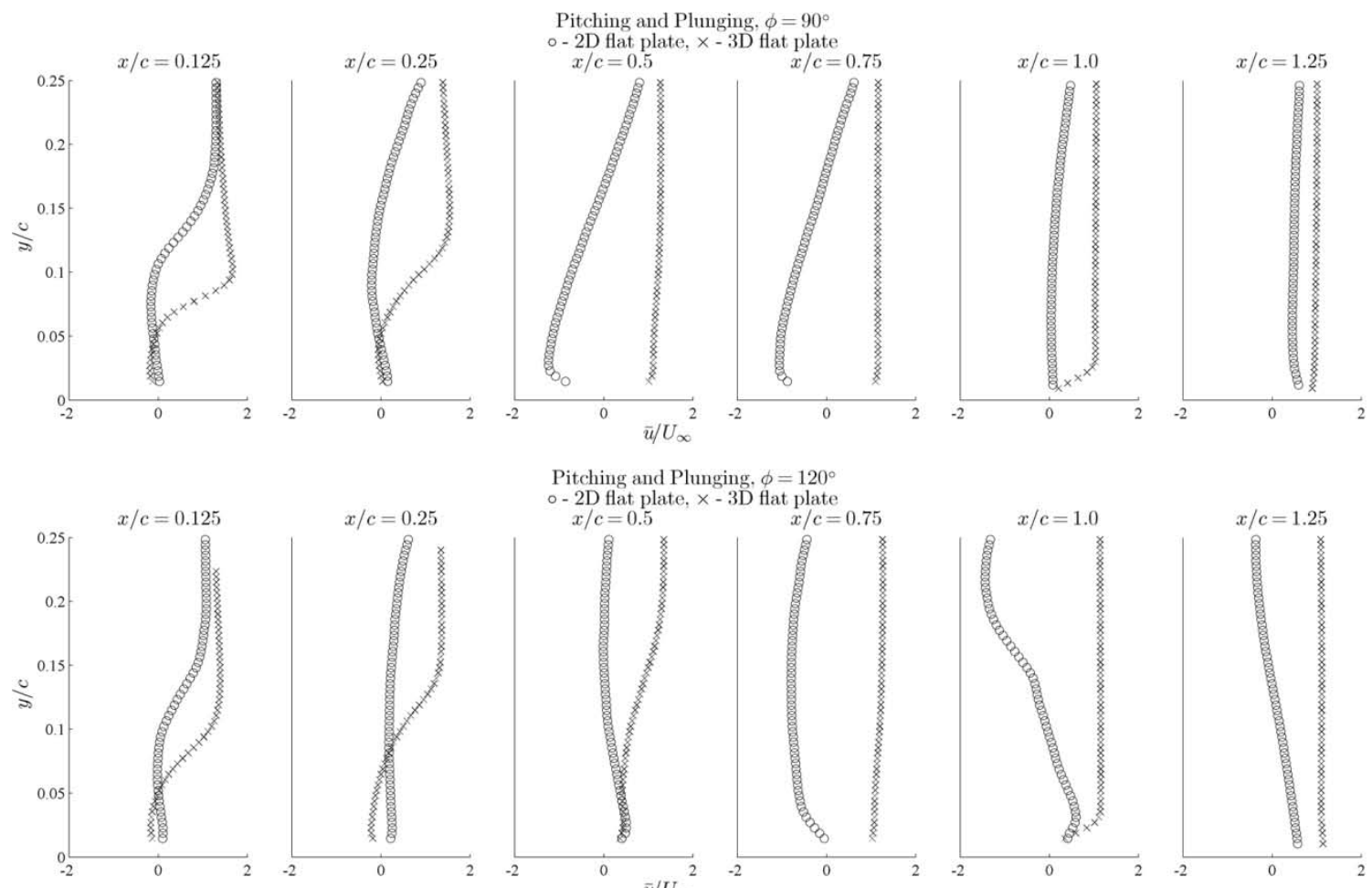

Pitching and Plunging, $\phi=120^{\circ}$ $0-2 \mathrm{D}$ flat plate, $x-3 \mathrm{D}$ flat plate
$x / c=0.5$
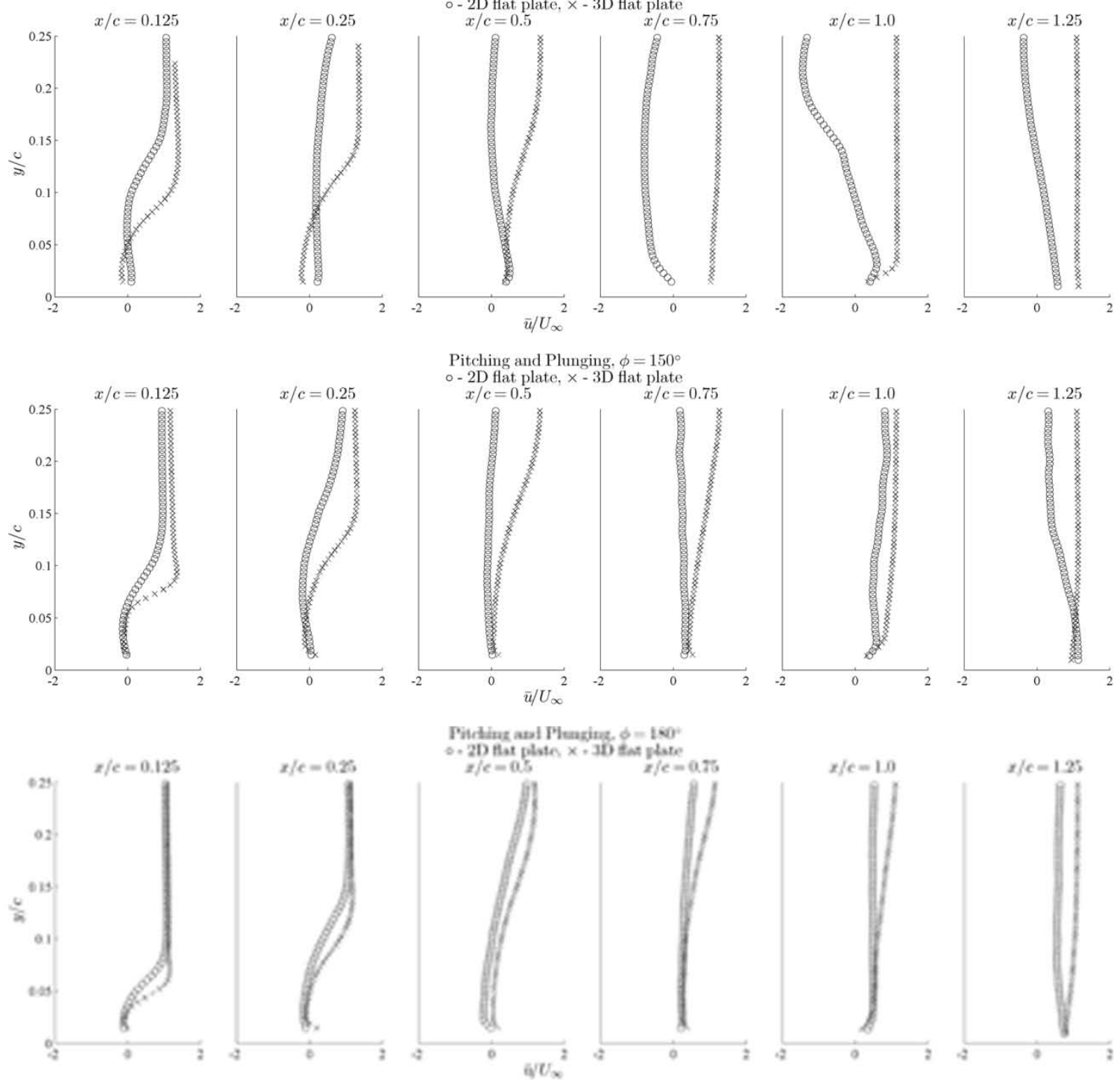

Figure 18. Plunging two- and three-dimensional flat plates at $\phi=90^{\circ}-180^{\circ}$, distributions of normalized $\bar{u}$ in the $y$-direction at selected chordwise stations $(x / c=0$ corresponds to the leading edge)

The normalized $\bar{u}$ distributions for the two plates are not comparable before large leading and trailing edge vortices are shed in the two-dimensional case. This poor comparison is present from $\phi=90^{\circ}-120^{\circ}$. While the 2D plate's normalized $\bar{u}$ distributions are dynamically changing for these phases, the 3D plate's normalized $\bar{u}$ 
distributions are comparatively static. The 3D plate over these phases shows the closed leading edge separation that does not extend beyond the half-chord.

\section{Extent of flow three-dimensionality}

The three-dimensionality of the flow over the both flat plates was investigated with flow visualization in the crossflow plane.

Dye streaks for the 2D plate showed minimal deviations fro $m$ the plane of the injector rake., but 3D plate results showed significant deviations from the plane of the injector. For the same phases of motion as the PIV, flow visualization of the spanwise flow is shown in Figure 19 for the pitching-and-plunging and pure-plunging 3D flat plate.

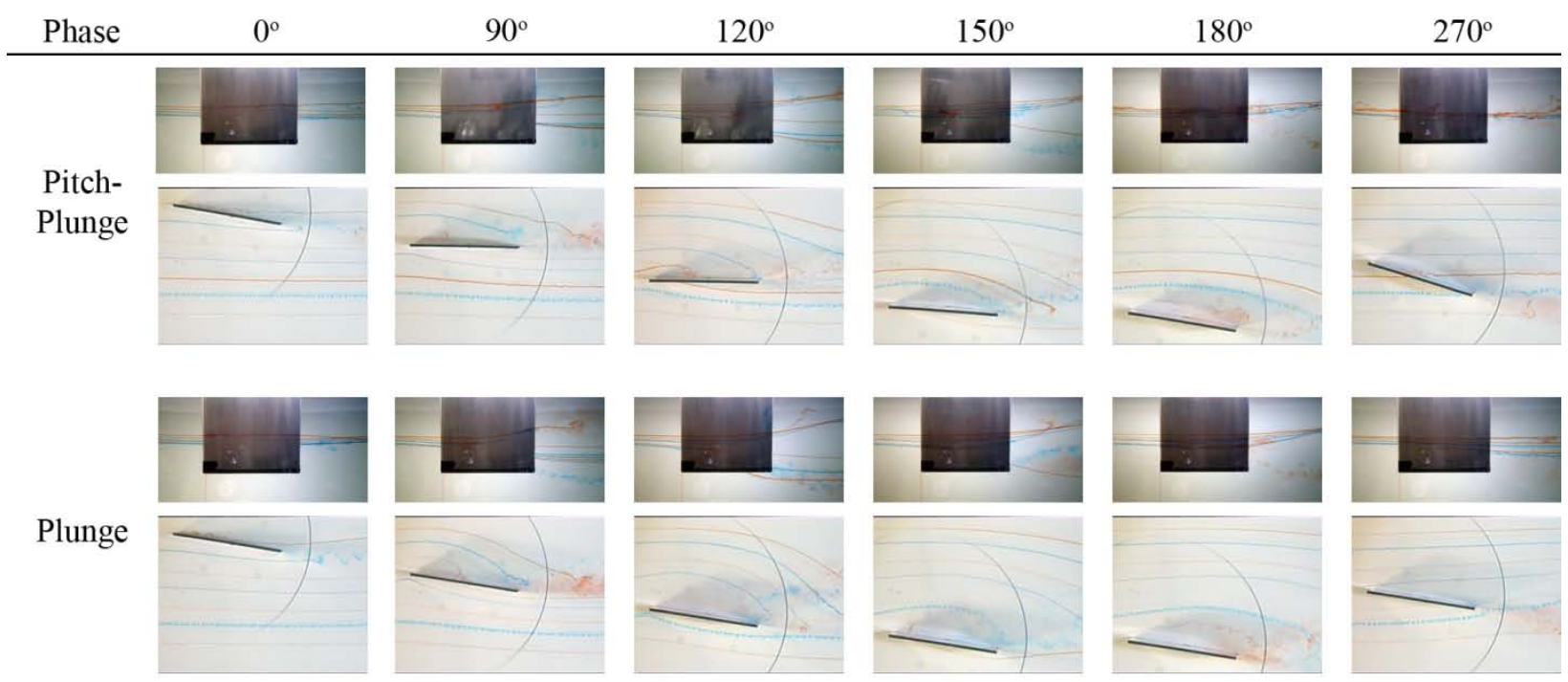

Figure 19. Flow visualization showing the spanwise flow for the 3D model in pitch-plunge and pure plunge. Notable spanwise flow occurs at the same range of phases for both motions, 90-180 degrees. Flow is from left-toright and the camera views the suction side of the flat plate.

The pitching and plunging $3 \mathrm{D}$ plate shows significant spanwise flow from $\phi=90^{\circ}-180^{\circ}$. The most significant three-dimensionality is caused by the rollup of the wing tip vortex. At $\phi=120^{\circ}$ and $150^{\circ}$ the wing tip vortex entrainment of dye from the pressure side of the flat plate can clearly be noted in Figure 19.

The plunging 3D plate has even significant spanwise flow associated with a wing tip vortex from $\phi=90^{\circ}-$ $180^{\circ}$. The wing tip vortex for the plunge motion causes more three-dimensionality at than the pitch-plunge motion. This is an excepted result since the large effective angle of attack time history for the plunge motion will cause a larger pressure differential between the pressure and suction side of the flat plate., as expected given the higher effective angle of attack at the plunge downstroke.

Significant three-dimensional flow was not observed in the closed leading edge separation, other than a gradual inboard deflection of the dye streaks. 


\section{Conclusions}

To generalize earlier work on wall-to-wall plunging and pitching-plunging plates to a plate of aspect ratio 2 , experiments at the University of Michigan's low-turbulence water tunnel used dye flow visualization in streamwise and crossflow planes, and particle image velocimetry in streamwise planes. Findings can be summarized as follows:

- For both the 2D and 3D plates, a reg ion of closed leading edge separation is present at $\boldsymbol{\phi}=\mathbf{9 0}^{\circ}$, halfway down the downstroke. For the 2D flat plate after $\boldsymbol{\phi}=\mathbf{9 0}^{\circ}$ the region of closed separation either forms a large leading edge vortex that sheds prior to the bottom of the downstroke (plunge) or the bottom of the downstroke is reached and the reduced effective angle of attack causes flow reattachment (pitch-plunge). For the 3D plate after $\boldsymbol{\phi}=\mathbf{9 0}^{\circ}$ the region of closed separation remains closed and confined to a reagion bounded by the leading edge and the half-chord.

- A discernable Reynolds number effect is present for the 2D plates in pure-plunge motion when comparing the Reynolds number 30,000 and 60,000. The pure-plunge motion makes a significantly larger excursion in effective angle of attack from the static stall angle of attack compared to the pitchplunge motion, resulting in a large leading edge vortex that forms and convects downstream. Presumably, this Reynolds number effect is absent in the pitch-plunge motion because the different flow physics as sociated with the large leading and trailing edge vortices created by the pure-plunge motion.

- The magnitude and physical extent of second order turbulence statistics for the low aspect ratio model are significantly smaller than the nominally $2 \mathrm{D}$ model

- Spanwise flow was observed by dye flow visualization for the 3D plate, but not for the 2D plate, thus providing confirmation for assumption of $2 \mathrm{D}$ flow for the latter. Spanwise flow for the $3 \mathrm{D}$ plate is associable with interaction with the wing tip vortex for both pitch-plunge and plunge motions. No appreciable spanwise flow was found in the closed leading edge separation.

- The University of Michigan and the AFRL PIV while qualitatively similar showed a greater chordwise separation for the AFRL PIV than the University of Michigan. Both data sets have closed separation through the downstroke with the exception being the plunging ARLF PIV at $\boldsymbol{\phi}=\mathbf{1 8 0}^{\circ}$ where open separation is observed. Without force measurement the significance of the flowfield differences cannot be assessed. 
A. Flow visualization and PIV from Baik, et al ${ }^{4}$ Appendix

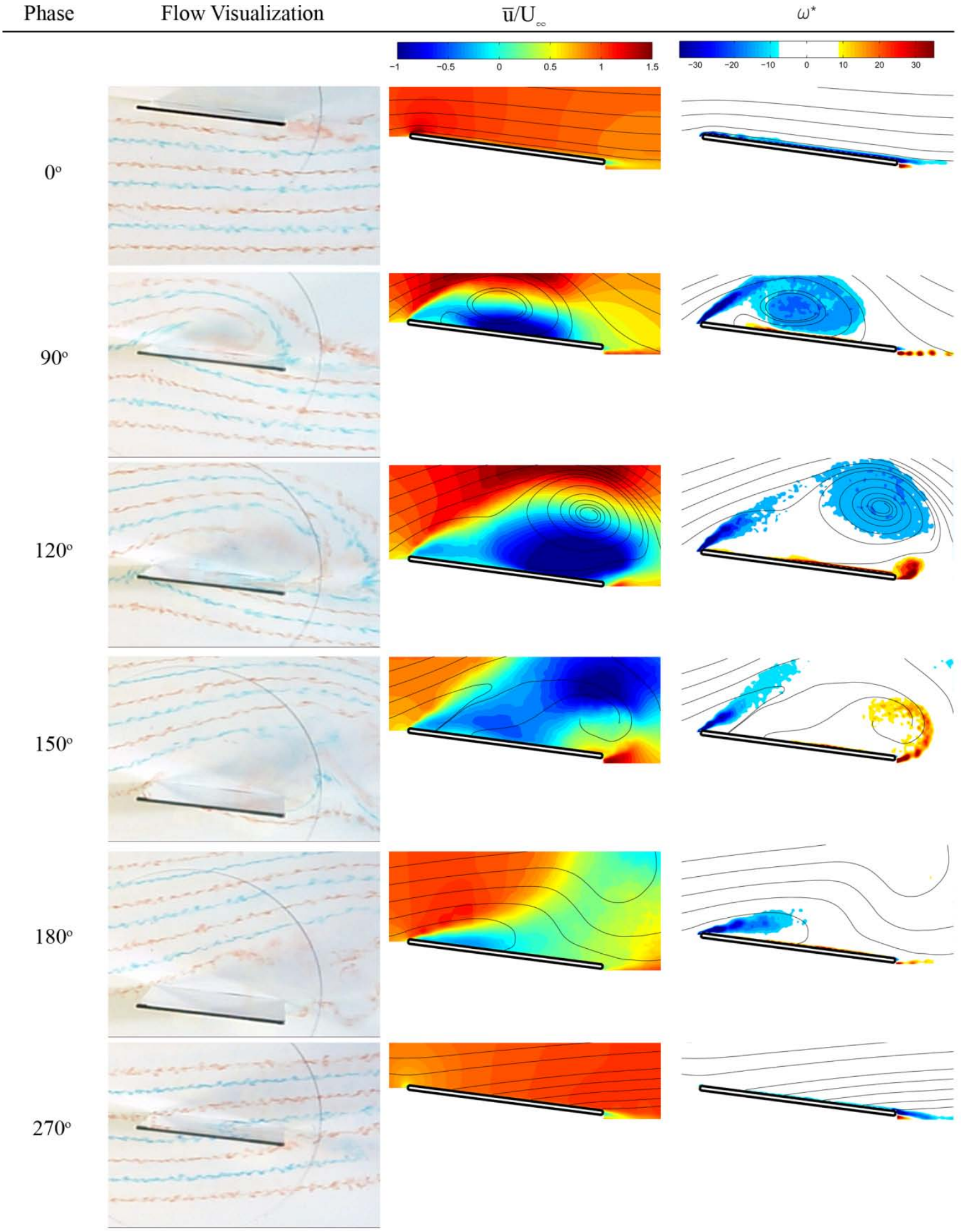

Figure A.1. Flow visualization, $\bar{u}$, and $\omega^{*}$ for the plunging 2 D flat plate at $\operatorname{Re}=60,000\left(\right.$ Baik, et al. $\left.{ }^{4}\right)$ 


\section{Acknowle dgements}

This work has been supported in part by the Air Force Office of Scientific Research's Multidisciplinary University Research Initiative.

\section{References}

${ }^{1}$ Shyy, W., Lian, Y., Tang, J., Viieru, D., \& Liu, H. (2008). Aerodynamics of Low Reynolds Number Flyers. Cambridge University Press.

${ }^{2}$ Ol, M., Bernal, L., Kang, C., and Shyy, W., "Shallow and deep dynamic stall for flapping low Reynolds number airfoils," Experiments in Fluids, Vol. 46, Nr. 5, 2009, pp. 883-901

${ }^{3}$ McCroskey, W.J, Carr, L.W., and McAlister, K.W., "Dynamic stall experiments on oscillating airfoils," Journal of Aircraft, Vol. 14, Nr. 1, 1976, pp. 57-63

${ }^{4}$ Baik, Y., Rausch, J.M., Bernal, L.P., and Ol, M.V., "Experimental Investigation of Pitching and Plunging Airfoils at Reynolds Number between $1 \times 10^{\wedge} 4$ and $6 \times 10^{\wedge} 4$," AIAA 2009-4030.

${ }^{5}$ Anderson, J. M., K. Streitlien, D. S. Barrett, and M. S. Triantafyllou. "Oscillating Foils of High Propulsive Efficiency." Journal of Fluid Mechanics 360 (1998): 41-72.

${ }^{6}$ Kang, C-K., Baik, Y.S., Bernal, L.P., Ol, M.V., and Shyy, W. "Fluid Dynamics of Pitching and Plunging Airfoils of Renolds Number between $1 \times 10^{\wedge} 4$ and 6x10^4." 47th Aerospace Sciences Meeting. Orlando: AIAA, 2009. 